ESAIM: M2AN 55 (2021) 329-356

https://doi.org/10.1051/m2an/2020069
ESAIM: Mathematical Modelling and Numerical Analysis

www.esaim-m2an.org

\title{
CENTRAL DISCONTINUOUS GALERKIN METHODS ON OVERLAPPING MESHES FOR WAVE EQUATIONS
}

\author{
Yong Liu ${ }^{1}$, Jianfang Lü,*, Chi-Wang Shu ${ }^{3}$ and Mengping Zhang ${ }^{4}$
}

\begin{abstract}
In this paper, we study the central discontinuous Galerkin (DG) method on overlapping meshes for second order wave equations. We consider the first order hyperbolic system, which is equivalent to the second order scalar equation, and construct the corresponding central DG scheme. We then provide the stability analysis and the optimal error estimates for the proposed central DG scheme for one- and multi-dimensional cases with piecewise $P^{k}$ elements. The optimal error estimates are valid for uniform Cartesian meshes and polynomials of arbitrary degree $k \geq 0$. In particular, we adopt the techniques in Liu et al. (SIAM J. Numer. Anal. 56 (2018) 520-541; ESAIM: M2AN 54 (2020) 705$726)$ and obtain the local projection that is crucial in deriving the optimal order of convergence. The construction of the projection here is more challenging since the unknowns are highly coupled in the proposed scheme. Dispersion analysis is performed on the proposed scheme for one dimensional problems, indicating that the numerical solution with $P^{1}$ elements reaches its minimum with a suitable parameter in the dissipation term. Several numerical examples including accuracy tests and long time simulation are presented to validate the theoretical results.
\end{abstract}

Mathematics Subject Classification. 65M60, 65M12, 65M15.

Received April 5, 2020. Accepted September 17, 2020.

\section{INTRODUCTION}

Wave propagation is a fundamental form of energy transmission, which arises in many fields of science, engineering and industry, such as geoscience, petroleum engineering, telecommunication, and the defense industry (see $[13,18]$ and the references therein). A vast amount of research can be found on the numerical approximation of wave problems. The commonly used numerical methods include finite difference, finite volume, spectral element and finite element methods, etc. (see e.g. $[12,14,15,19]$ ). Among those numerical methods, we confine our attention to the discontinuous Galerkin (DG) methods in this paper. DG methods are a class of finite element methods using discontinuous basis functions, which are usually chosen as piecewise polynomials, but

Keywords and phrases. Optimal error estimates, central DG method, second order wave equation, dispersion analysis.

1 Hua Loo-Keng Center for Mathematical Sciences, Academy of Mathematics and Systems Science, Chinese Academy of Sciences, Beijing 100190, P.R. China.

2 South China Research Center for Applied Mathematics and Interdisciplinary Studies, South China Normal University, Canton 510631, Guangdong, P.R. China.

3 Division of Applied Mathematics, Brown University, Providence, RI 02912, USA.

4 School of Mathematical Sciences, University of Science and Technology of China, Hefei 230026, Anhui, P.R. China.

*Corresponding author: jflu@m.scnu.edu.cn 
could also be chosen as other types of functions to suit specific needs. The first DG method was developed to solve a steady transport equation in [25], and later Cockburn et al. applied the DG discretization in space, coupled with explicit Runge-Kutta time discretization, for solving the hyperbolic conservation laws successfully [6-10]. Since then, the DG method has attracted more and more attention, and found broad applications in various areas such as aero-acoustics, gas dynamics, weather forecasting, oceanography, electro-magnetism, etc. The DG method has many advantages such as allowing triangulations with hanging nodes, the extremely local data structure, high efficiency in parallel computation, and the ability to easily accommodate arbitrary $h-p$ adaptivity, etc.

In this paper, we propose a central discontinuous Galerkin (CDG) scheme for solving the following two way wave equation

$$
\left\{\begin{array}{l}
p_{t}=c \nabla \cdot \boldsymbol{q}+f(p, \boldsymbol{q}, \boldsymbol{x}, t) \quad \text { in } \Omega, \quad t>0 \\
\boldsymbol{q}_{t}=c \nabla p+\boldsymbol{g}(p, \boldsymbol{q}, \boldsymbol{x}, t) \quad \text { in } \Omega, \quad t>0
\end{array}\right.
$$

where $\Omega \subset \mathbb{R}^{d}$ is some bounded domain, $d=1,2,3$, and $p=p(\boldsymbol{x}, t)$ and $\boldsymbol{q}=\boldsymbol{q}(\boldsymbol{x}, t) \in \mathbb{R}^{d}$ are unknown functions, and the source terms $f(p, \boldsymbol{q}, \boldsymbol{x}, t) \in \mathbb{R}$ and $\boldsymbol{g}(p, \boldsymbol{q}, \boldsymbol{x}, t) \in \mathbb{R}^{d}$. The system (1.1) without source terms is equivalent to the acoustic wave equation

$$
p_{t t}=c^{2} \Delta p
$$

where $\Delta$ is the Laplace operator, $p$ is the acoustic pressure (the local deviation from the ambient pressure), and $c$ is the speed of sound. For simplicity, in the remainder of the paper we will only consider the system (1.1) without source terms, i.e. $f(p, \boldsymbol{q}, \boldsymbol{x}, t)=0, \boldsymbol{g}(p, \boldsymbol{q}, \boldsymbol{x}, t)=\mathbf{0}$, as all results also hold for the general case with the source terms depending linearly on $p$ and $\boldsymbol{q}$. That is, we will only consider the following system:

$$
\begin{cases}p_{t}=c \nabla \cdot \boldsymbol{q} \text { in } \Omega, \quad t>0 \\ \boldsymbol{q}_{t}=c \nabla p \quad \text { in } \Omega, & t>0 .\end{cases}
$$

The central DG method was first introduced by Liu et al. [20]. In order to avoid Riemann solvers in constructing the numerical fluxes at the interfaces of the elements, in [20] the authors computed two numerical solutions on the overlapping meshes. Therefore, one advantage of the central DG method is the avoidance of the possibly complicated construction of the numerical fluxes. Another advantage is that it allows a larger time step (proportional to $O(h / k)$ where $h$ is the spatial mesh size and $k$ is the polynomial degree) than that of the regular DG method (which has a time step proportional to $O\left(h / k^{2}\right)$ ), particularly for higher order of spatial accuracy [26]. Liu et al. [21] provided $L^{2}$ stability analysis and suboptimal error estimates for linear hyperbolic equations. Later Liu et al. in [22] used the shifting technique to construct a special projection to obtain optimal error estimates of the central DG methods for linear hyperbolic equations. In [23] they continue to use this technique to obtain optimal error estimates of the DG methods on Cartesian meshes using $P^{k}$ element space, which is the space of piecewise polynomials with degree at most $k$ in each element. Recently, the shifting technique was adopted in the study of optimal convergence and superconvergence of semi-Lagrangian DG methods in [30].

To solve the second order wave equation numerically, one approach is to construct the numerical scheme directly such as the symmetric interior penalty DG method [17]. Another way is to introduce the auxiliary variables and rewrite the wave equation into a first order system, then construct the corresponding numerical schemes, see e.g. $[1,3,4,27]$. In this paper, we propose a central DG scheme for solving the first order system (1.3). Our main contribution in this paper is that we provide the $L^{2}$ stability and the optimal error estimates for the proposed central DG scheme using $P^{k}$ elements. The proof of optimal convergence results is valid for uniform meshes and for polynomials of arbitrary degree $k \geq 0$. Though we perform the analysis in one and two dimensions, the analysis can be extended to higher dimensional problems without any essential difficulties. For the multidimensional problems on Cartesian meshes, the optimal error estimates are usually based on the use of the $Q^{k}$ element space, which is the tensor product of the one dimensional finite element spaces $[11,24,28]$. Thanks 
to the shifting technique in [22], we are able to construct a special local coupled projection on the overlapping meshes, thus we obtain the optimal error estimates with the use of $P^{k}$ elements. The local coupled projection can eliminate the space-discrete terms when $(p, \boldsymbol{q})$ are both in the $P^{k+1}$ space. This superconvergence result leads to the derivation of optimal convergence rate. To the best knowledge of the authors, this is the first work obtaining optimal convergence rate when using the $P^{k}$ elements on overlapping meshes for multidimensional problems. We also provide a dispersion analysis for the proposed scheme in one dimension based on the Fourier analysis.

The organization of this paper is as follows. In Section 2, we propose the central DG scheme for the wave equation (1.3). In Section 3, we prove the $L^{2}$ stability and optimal error estimates for one- and multi-dimensional problems. The dispersion analysis for one dimensional problems is also provided, with the $P^{k}$ elements for $k=0,1,2$. Numerical experiments are provided to verify our theoretical results in Section 4. In Section 5, we give a few concluding remarks and perspectives for future work. Some technical proofs of the lemmas are provided in the Appendix A.

\section{The CEntral DG SCHEME}

In this section, we present the central DG scheme for (1.3). First, we multiply the first and second equations with functions $\varphi$ and $\psi$, respectively, and perform integration by parts on an open and bounded subset $K \subseteq \Omega$, to obtain the weak formulation as follows

$$
\begin{aligned}
\int_{K} p_{t} \varphi \mathrm{d} \boldsymbol{x} & =-\int_{K} c \boldsymbol{q} \cdot \nabla \varphi \mathrm{d} \boldsymbol{x}+\int_{\partial K} c \boldsymbol{q} \cdot \boldsymbol{n}_{K} \varphi \mathrm{d} s, \\
\int_{K} \boldsymbol{q}_{t} \cdot \boldsymbol{\psi} \mathrm{d} \boldsymbol{x} & =-\int_{K} c p \nabla \cdot \boldsymbol{\psi} \mathrm{d} \boldsymbol{x}+\int_{\partial K} c p \boldsymbol{n}_{K} \cdot \boldsymbol{\psi} \mathrm{d} s,
\end{aligned}
$$

where $\boldsymbol{n}_{K}$ is the unit outward normal to $\partial K$. Now we define the central DG scheme from the variational form (2.1). To this end, we first take the partition of the domain. Assume we have two kinds of partitions $\mathcal{T}_{h}$ and $\mathcal{T}_{h}^{\prime}$ on $\Omega$. The partitions $\mathcal{T}_{h}$ and $\mathcal{T}_{h}^{\prime}$ are overlapped and are usually chosen as the overlapping Cartesian meshes. For general cases, we refer to [29]. A two dimensional overlapping Cartesian mesh is shown in Figure 1.

Now we introduce the finite element spaces associated with these two partitions.

$$
\begin{aligned}
V_{h} & :=\left\{\varphi_{h} \in L^{2}(\Omega):\left.\varphi_{h}\right|_{K} \in P^{k}(K) \quad \forall K \in \mathcal{T}_{h}\right\}, \\
W_{h} & :=\left\{\psi_{h} \in L^{2}(\Omega):\left.\psi_{h}\right|_{K^{\prime}} \in P^{k}\left(K^{\prime}\right) \quad \forall K^{\prime} \in \mathcal{T}_{h}^{\prime}\right\} .
\end{aligned}
$$

The central DG formulation is defined as follows: Find $p_{h} \in V_{h}, \boldsymbol{q}_{h} \in\left[V_{h}\right]^{d}$ and $r_{h} \in W_{h}, \boldsymbol{s}_{h} \in\left[W_{h}\right]^{d}$ such that for all $K \in \mathcal{T}_{h}$ and $K^{\prime} \in \mathcal{T}_{h}^{\prime}$ we have

$$
\begin{aligned}
\int_{K}\left(p_{h}\right)_{t} \varphi_{h} \mathrm{~d} \boldsymbol{x}= & -\int_{K} c \boldsymbol{s}_{h} \cdot \nabla \varphi_{h} \mathrm{~d} \boldsymbol{x}+\int_{\partial K} c \boldsymbol{s}_{h} \cdot \boldsymbol{n}_{K} \varphi_{h} \mathrm{~d} s \\
& +\frac{1}{\tau_{\max }} \int_{K}\left(r_{h}-p_{h}\right) \varphi_{h} \mathrm{~d} \boldsymbol{x}, \quad \forall \varphi_{h} \in V_{h}, \\
\int_{K}\left(\boldsymbol{q}_{h}\right)_{t} \cdot \overline{\boldsymbol{\varphi}}_{h} \mathrm{~d} \boldsymbol{x}= & -\int_{K} c r_{h} \nabla \cdot \overline{\boldsymbol{\varphi}}_{h} \mathrm{~d} \boldsymbol{x}+\int_{\partial K} c r_{h} \boldsymbol{n}_{K} \cdot \overline{\boldsymbol{\varphi}}_{h} \mathrm{~d} s \\
& +\frac{1}{\tau_{\max }} \int_{K}\left(\boldsymbol{s}_{h}-\boldsymbol{q}_{h}\right) \cdot \overline{\boldsymbol{\varphi}}_{h} \mathrm{~d} \boldsymbol{x}, \quad \forall \overline{\boldsymbol{\varphi}}_{h} \in\left[V_{h}\right]^{d}, \\
\int_{K^{\prime}}\left(r_{h}\right)_{t} \psi_{h} \mathrm{~d} \boldsymbol{x}= & -\int_{K^{\prime}} c \boldsymbol{q}_{h} \cdot \nabla \psi_{h} \mathrm{~d} \boldsymbol{x}+\int_{\partial K^{\prime}} c \boldsymbol{q}_{h} \cdot \boldsymbol{n}_{K^{\prime}} \psi_{h} \mathrm{~d} s \\
& +\frac{1}{\tau_{\max }} \int_{K^{\prime}}\left(p_{h}-r_{h}\right) \psi_{h} \mathrm{~d} \boldsymbol{x}, \quad \forall \psi_{h} \in W_{h},
\end{aligned}
$$




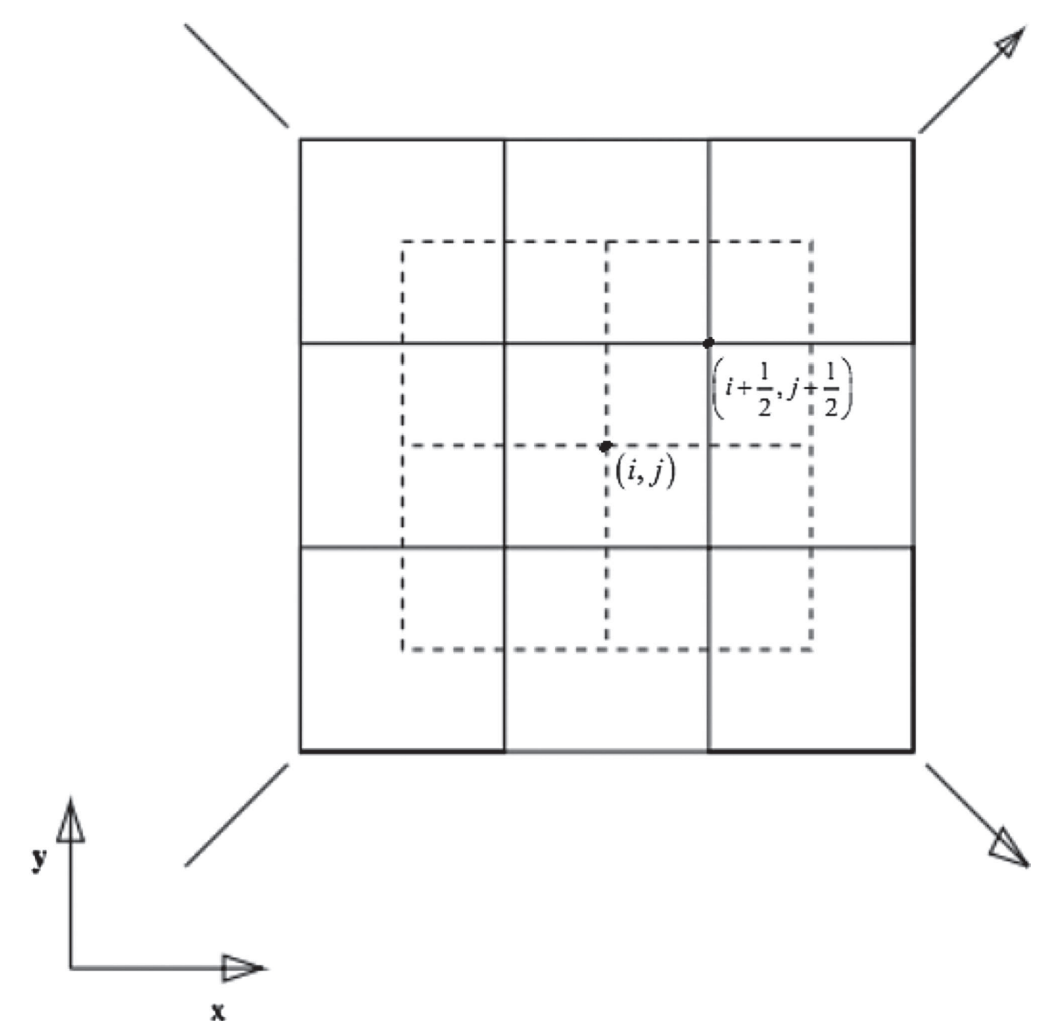

Figure 1. 2D overlapping cells, the first mesh is formed by solid lines, and the dual mesh is formed by dashed lines.

$$
\begin{aligned}
\int_{K^{\prime}}\left(\boldsymbol{s}_{h}\right)_{t} \cdot \overline{\boldsymbol{\psi}}_{h} \mathrm{~d} \boldsymbol{x}= & -\int_{K^{\prime}} c p_{h} \nabla \cdot \overline{\boldsymbol{\psi}}_{h} \mathrm{~d} \boldsymbol{x}+\int_{\partial K^{\prime}} c p_{h} \boldsymbol{n}_{K^{\prime}} \cdot \overline{\boldsymbol{\psi}}_{h} \mathrm{~d} s \\
& +\frac{1}{\tau_{\max }} \int_{K^{\prime}}\left(\boldsymbol{q}_{h}-\boldsymbol{s}_{h}\right) \cdot \overline{\boldsymbol{\psi}}_{h} \mathrm{~d} \boldsymbol{x}, \quad \forall \overline{\boldsymbol{\psi}}_{h} \in\left[W_{h}\right]^{d},
\end{aligned}
$$

where $\left[V_{h}\right]^{d}=\overbrace{V_{h} \times \ldots \times V_{h}}^{d}$, and $\tau_{\max }$ is an upper bound for the time step size due to the CFL restriction, that is, $\tau_{\max }=\alpha h$ with a given constant CFL number $\alpha>0$ dictated by stability. $p_{h}, r_{h}$ are the approximations to the solution $p$, and $\boldsymbol{q}_{h}, \boldsymbol{s}_{h}$ are the approximations to the solution $\boldsymbol{q}$. The initial data is obtained by the standard $L^{2}$ projection, that is, $p_{h}(\cdot, 0)=\mathbb{P}_{h} p_{0}, r_{h}(\cdot, 0)=\mathbb{Q}_{h} p_{0}, q_{h}^{i}(\cdot, 0)=\mathbb{P}_{h} q_{0}^{i}$ and $s_{h}^{i}(\cdot, 0)=\mathbb{Q}_{h} q_{0}^{i}, i=1, \ldots, d$, where $q_{0}^{i}$ is the $i$-th argument of $\boldsymbol{q}_{0}$, and $q_{h}^{i}$ and $s_{h}^{i}$ are the $i$-th arguments of $\boldsymbol{q}_{h}$ and $\boldsymbol{s}_{h}$, respectively. The $L^{2}$ projections $\mathbb{P}_{h}$ and $\mathbb{Q}_{h}$ are defined as follows. For any function $u$, we have $\mathbb{P}_{h} u \in P^{k}(K)$ and $\mathbb{Q}_{h} u \in P^{k}\left(K^{\prime}\right)$ satisfying

$$
\begin{gathered}
\int_{K}\left(\mathbb{P}_{h} u-u\right) \varphi_{h} \mathrm{~d} \boldsymbol{x}=0, \quad \forall \varphi_{h} \in P^{k}(K), \forall K \in \mathcal{T}_{h}, \\
\int_{K^{\prime}}\left(\mathbb{Q}_{h} u-u\right) \bar{\varphi}_{h} \mathrm{~d} \boldsymbol{x}=0, \quad \forall \bar{\varphi}_{h} \in P^{k}\left(K^{\prime}\right), \forall K^{\prime} \in \mathcal{T}_{h}^{\prime} .
\end{gathered}
$$

From Theorem 3.1 .5 in [5] we have

$$
\begin{aligned}
\left\|p_{h}(\cdot, 0)-p_{0}\right\|+\left\|r_{h}(\cdot, 0)-p_{0}\right\| & \lesssim h^{k+1}\left\|p_{0}\right\|_{k+1}, \\
\left\|\boldsymbol{q}_{h}(\cdot, 0)-\boldsymbol{q}_{0}\right\|+\left\|\boldsymbol{s}_{h}(\cdot, 0)-\boldsymbol{q}_{0}\right\| & \lesssim h^{k+1}\left\|\boldsymbol{q}_{0}\right\|_{k+1},
\end{aligned}
$$


where the unmarked norm $\|\cdot\|$ denotes the standard $L^{2}$ norm on $\Omega$, and $\|\cdot\|_{k+1}$ is the standard norm in the Sobolev space $W^{k+1,2}(\Omega)$.

\section{Analysis of the Central DG schemes}

In this section, we analyze the proposed central DG scheme (2.3) for equation (1.3). We present the $L^{2}$ stability, and the a priori optimal error estimates for one and two dimensional problems only. As we can see, the techniques we take are very general and can be extended to higher dimensional problems without any difficulties. We also provide a dispersion analysis for one dimensional problems. Before we proceed, we introduce some standard Sobolev spaces notations. For any integer $m>0$, let $W^{m, p}(D)$ be the standard Sobolev spaces on sub-domain $D \subseteq \Omega$ equipped with the norm $\|\cdot\|_{m, p, D}$ and semi-norm $|\cdot|_{m, p, D}$. When $D=\Omega$, we omit the index $D$; and if $p=2$, we set $W^{m, p}(D)=H^{m}(D),\|\cdot\|_{m, p, D}=\|\cdot\|_{m, D}$, and $|\cdot|_{m, p, D}=|\cdot|_{m, D}$. An unmarked norm $\|\cdot\|$ denotes the standard $L^{2}$ norm.

\subsection{Analysis of the central DG method in one dimension}

For one dimensional case, the equation (1.3) becomes

$$
\begin{cases}p_{t}=c q_{x}, & x \in \Omega, t>0, \\ q_{t}=c p_{x}, & x \in \Omega, t>0, \\ p(x, 0)=p_{0}(x), & q(x, 0)=q_{0}(x),\end{cases}
$$

with periodic boundary condition. Without loss of generality, we assume that $c=-1$ and $\Omega=[0,1]$. Let $\left\{x_{j}\right\}$ be a partition of $[0,1]$ with $h_{j+\frac{1}{2}}=x_{j+1}-x_{j}$ and $h=\max _{j} h_{j+\frac{1}{2}}$. Denote $x_{j+\frac{1}{2}}=\left(x_{j+1}+x_{j}\right) / 2, I_{j}=\left(x_{j+\frac{1}{2}}, x_{j-\frac{1}{2}}\right)$, and $I_{j+\frac{1}{2}}=\left(x_{j}, x_{j+1}\right)$. Then we have the corresponding finite element spaces as follows.

$$
\begin{aligned}
V_{h} & :=\left\{\varphi_{h} \in L^{2}(\Omega):\left.\varphi_{h}\right|_{I_{j}} \in P^{k}\left(I_{j}\right), \quad \forall j\right\}, \\
W_{h} & :=\left\{\psi_{h} \in L^{2}(\Omega):\left.\psi_{h}\right|_{I_{j+\frac{1}{2}}} \in P^{k}\left(I_{j+\frac{1}{2}}\right), \quad \forall j\right\} .
\end{aligned}
$$

$V_{h}$ is the set of piecewise polynomials of degree at most $k$ over the subintervals $\left\{I_{j}\right\}$ with no continuity assumed across the subinterval boundaries. Likewise, $W_{h}$ is the set of piecewise polynomials of degree at most $k$ over the subintervals $I_{j+\frac{1}{2}}$ with no continuity assumed across the subinterval boundaries.

The semidiscrete version of the central DG scheme for solving (3.1) is defined as follows: Find $p_{h}(\cdot, t), q_{h}(\cdot, t) \in V_{h}$ and $r_{h}(\cdot, t), s_{h}(\cdot, t) \in W_{h}$ such that for any $\varphi_{h}, \bar{\varphi}_{h} \in V_{h}$ and $\psi_{h}, \bar{\psi}_{h} \in W_{h}$, we have

$$
\begin{gathered}
\int_{I_{j}}\left(p_{h}\right)_{t} \varphi_{h} \mathrm{~d} x=B_{1}\left(r_{h}, p_{h}, s_{h} ; \varphi_{h}\right)_{j}, \\
\int_{I_{j}}\left(q_{h}\right)_{t} \bar{\varphi}_{h} \mathrm{~d} x=B_{1}\left(s_{h}, q_{h}, r_{h} ; \bar{\varphi}_{h}\right)_{j}, \\
\int_{I_{j+\frac{1}{2}}}\left(r_{h}\right)_{t} \psi_{h} \mathrm{~d} x=B_{2}\left(p_{h}, r_{h}, q_{h} ; \psi_{h}\right)_{j+\frac{1}{2}}, \\
\int_{I_{j+\frac{1}{2}}}\left(s_{h}\right)_{t} \bar{\psi}_{h} \mathrm{~d} x=B_{2}\left(q_{h}, s_{h}, p_{h} ; \bar{\psi}_{h}\right)_{j+\frac{1}{2}},
\end{gathered}
$$


where

$$
\begin{aligned}
B_{1}\left(r_{h}, p_{h}, s_{h} ; \varphi_{h}\right)_{j}= & \frac{1}{\tau_{\max }} \int_{I_{j}}\left(r_{h}-p_{h}\right) \varphi_{h} \mathrm{~d} x+\int_{I_{j}} s_{h}\left(\varphi_{h}\right)_{x} \mathrm{~d} x \\
& -s_{h}\left(x_{j+\frac{1}{2}}, t\right) \varphi_{h}\left(x_{j+\frac{1}{2}}^{-}\right)+s_{h}\left(x_{j-\frac{1}{2}}, t\right) \varphi_{h}\left(x_{j-\frac{1}{2}}^{+}\right), \\
B_{2}\left(p_{h}, r_{h}, q_{h} ; \psi_{h}\right)_{j+\frac{1}{2}}= & \frac{1}{\tau_{\max }} \int_{I_{j+\frac{1}{2}}}\left(p_{h}-r_{h}\right) \psi_{h} \mathrm{~d} x+\int_{I_{j+\frac{1}{2}}} q_{h}\left(\psi_{h}\right)_{x} \mathrm{~d} x \\
& -q_{h}\left(x_{j+1}, t\right) \psi_{h}\left(x_{j+1}^{-}\right)+q_{h}\left(x_{j}, t\right) \psi_{h}\left(x_{j}^{+}\right) .
\end{aligned}
$$

In this subsection, we study the $L^{2}$ stability of the central DG scheme on overlapping cells (3.2) for the equation (3.1), and then we provide an $L^{2}$ a priori optimal error estimates for smooth solutions.

Theorem 3.1 ( $L^{2}$ stability). The numerical solution $p_{h}, r_{h}, q_{h}$ and $s_{h}$ of the central DG scheme (3.2) for the equation (3.1) satisfies the following $L^{2}$ stability condition

$$
\frac{1}{2} \frac{\mathrm{d}}{\mathrm{d} t} \int_{0}^{1}\left(\left(p_{h}\right)^{2}+\left(q_{h}\right)^{2}+\left(r_{h}\right)^{2}+\left(s_{h}\right)^{2}\right) \mathrm{d} x+\frac{1}{\tau_{\max }} \int_{0}^{1}\left(\left(p_{h}-r_{h}\right)^{2}+\left(q_{h}-s_{h}\right)^{2}\right) \mathrm{d} x=0 .
$$

Proof. Taking the test functions $\varphi_{h}=p_{h}, \psi_{h}=r_{h}, \bar{\varphi}_{h}=q_{h}$ and $\bar{\psi}_{h}=s_{h}$ in (3.2) respectively, then summing it up over $j$, with the periodic boundary condition we have

$$
\begin{aligned}
\frac{1}{2} \frac{\mathrm{d}}{\mathrm{d} t} \int_{0}^{1}\left(\left(p_{h}\right)^{2}+\left(q_{h}\right)^{2}+\left(r_{h}\right)^{2}+\left(s_{h}\right)^{2}\right) \mathrm{d} x \\
=\sum_{j} \frac{1}{\tau_{\max }}\left(\int_{x_{j-\frac{1}{2}}}^{x_{j+\frac{1}{2}}}\left(r_{h}-p_{h}\right) p_{h} \mathrm{~d} x+\int_{x_{j}}^{x_{j+1}}\left(p_{h}-r_{h}\right) r_{h} \mathrm{~d} x\right) \\
\quad+\frac{1}{\tau_{\max }}\left(\int_{x_{j-\frac{1}{2}}}^{x_{j+\frac{1}{2}}}\left(s_{h}-q_{h}\right) q_{h} \mathrm{~d} x+\int_{x_{j}}^{x_{j+1}}\left(q_{h}-s_{h}\right) s_{h} \mathrm{~d} x\right) \\
\quad+\int_{I_{j}} s_{h} \partial_{x} p_{h} \mathrm{~d} x+\int_{I_{j+\frac{1}{2}}} p_{h} \partial_{x} s_{h} \mathrm{~d} x+\int_{I_{j}} r_{h} \partial_{x} q_{h} \mathrm{~d} x+\int_{I_{j+\frac{1}{2}}} q_{h} \partial_{x} r_{h} \mathrm{~d} x \\
\quad+s_{h}\left(x_{j-\frac{1}{2}}\right) p_{h}\left(x_{j-\frac{1}{2}}^{+}\right)-s_{h}\left(x_{j}^{-}\right) p_{h}\left(x_{j}\right)-s_{h}\left(x_{j+\frac{1}{2}}\right) p_{h}\left(x_{j+\frac{1}{2}}^{-}\right)+s_{h}\left(x_{j}^{+}\right) p_{h}\left(x_{j}\right) \\
\quad+r_{h}\left(x_{j-\frac{1}{2}}\right) q_{h}\left(x_{j-\frac{1}{2}}^{+}\right)-r_{h}\left(x_{j}^{-}\right) q_{h}\left(x_{j}\right)-r_{h}\left(x_{j+\frac{1}{2}}\right) q_{h}\left(x_{j+\frac{1}{2}}^{-}\right)+r_{h}\left(x_{j}^{+}\right) q_{h}\left(x_{j}\right) \\
=-\frac{1}{\tau_{\max }} \int_{0}^{1}\left(p_{h}-r_{h}\right)^{2} \mathrm{~d} x-\frac{1}{\tau_{\max }} \int_{0}^{1}\left(q_{h}-s_{h}\right)^{2} \mathrm{~d} x .
\end{aligned}
$$

To prove the optimal error estimates of the central DG scheme, we first introduce some notations. Throughout this paper, $A \lesssim B$ denotes that $A$ can be bounded by $B$ multiplied by a constant independent of the mesh size $h$. Define

$$
\begin{aligned}
& A_{j}\left(p_{h}, r_{h}, q_{h}, s_{h} ; \varphi_{h}, \psi_{h}, \bar{\varphi}_{h}, \bar{\psi}_{h}\right) \\
& =\int_{I_{j}} \partial_{t} p_{h} \varphi_{h} \mathrm{~d} x+\int_{I_{j}} \partial_{t} q_{h} \bar{\varphi}_{h} \mathrm{~d} x+\int_{I_{j+\frac{1}{2}}} \partial_{t} r_{h} \psi_{h} \mathrm{~d} x+\int_{I_{j+\frac{1}{2}}} \partial_{t} s_{h} \bar{\psi}_{h} \mathrm{~d} x \\
& \quad-B_{1}\left(r_{h}, p_{h}, s_{h} ; \varphi_{h}\right)_{j}-B_{1}\left(s_{h}, q_{h}, r_{h} ; \bar{\varphi}_{h}\right)_{j} \\
& \quad-B_{2}\left(p_{h}, r_{h}, q_{h} ; \psi_{h}\right)_{j+\frac{1}{2}}-B_{2}\left(q_{h}, s_{h}, p_{h} ; \bar{\psi}_{h}\right)_{j+\frac{1}{2}} .
\end{aligned}
$$


Clearly, $\forall j$ and $\forall \varphi_{h}, \bar{\varphi}_{h} \in V_{h}, \psi_{h}, \bar{\psi}_{h} \in W_{h}$ we have

$$
A_{j}\left(p_{h}, r_{h}, q_{h}, s_{h} ; \varphi_{h}, \psi_{h}, \bar{\varphi}_{h}, \bar{\psi}_{h}\right)=0 .
$$

Due to the consistency of the scheme (3.2), the exact solutions $p, q$ also satisfy

$$
A_{j}\left(p, p, q, q ; \varphi_{h}, \psi_{h}, \bar{\varphi}_{h}, \bar{\psi}_{h}\right)=0, \quad \forall j \text { and } \forall \varphi_{h}, \bar{\varphi}_{h} \in V_{h}, \psi_{h}, \bar{\psi}_{h} \in W_{h} .
$$

Subtracting (3.6) from (3.7), we obtain the error equation

$$
A_{j}\left(p-p_{h}, p-r_{h}, q-q_{h}, q-s_{h} ; \varphi_{h}, \psi_{h}, \bar{\varphi}_{h}, \bar{\psi}_{h}\right)=0, \quad \forall j \text { and } \forall \varphi_{h}, \bar{\varphi}_{h} \in V_{h}, \psi_{h}, \bar{\psi}_{h} \in W_{h} .
$$

We also recall the following basic facts. For any function $w_{h} \in V_{h}$ or $W_{h}$, the following inverse inequalities hold from Theorem 3.2.6 in [5]:

$$
\left\|\left(w_{h}\right)_{x}\right\| \lesssim h^{-1}\left\|w_{h}\right\|, \quad\left\|w_{h}\right\|_{\infty} \lesssim h^{-\frac{1}{2}}\left\|w_{h}\right\|, \quad\left\|w_{h}\right\|_{\Gamma_{h}} \lesssim h^{-\frac{1}{2}}\left\|w_{h}\right\|,
$$

where $\Gamma_{h}$ denotes the set of boundary points of all elements $I_{j}$ or $I_{j+\frac{1}{2}}$ respectively, and the norm $\|\cdot\|_{\Gamma_{h}}$ is the standard $L^{2}$ norm. We now define the special local coupled projection. For any function $p, q \in H^{1}(\Omega)$, define the following coupled projection $\mathbb{P}_{h}^{\star}(p, q):=\left(\mathbb{P}_{h}^{1, \star} p, \mathbb{P}_{h}^{2, \star} q\right) \in\left[V_{h}\right]^{2}$ such that

$$
\begin{aligned}
& \int_{I_{j}} \mathbb{P}_{h}^{1, \star} p(x) \mathrm{d} x=\int_{I_{j}} p(x) \mathrm{d} x, \\
& \int_{I_{j}} \mathbb{P}_{h}^{2, \star} q(x) \mathrm{d} x=\int_{I_{j}} q(x) \mathrm{d} x, \\
& \widetilde{P_{h}}\left(\mathbb{P}_{h}^{1, \star} p(x), \mathbb{P}_{h}^{2, \star} q(x) ; \varphi_{h}\right)_{j}=\widetilde{P_{h}}\left(p(x), q(x) ; \varphi_{h}\right)_{j} \quad \forall \varphi_{h} \in P^{k}\left(I_{j}\right), \\
& \widetilde{P_{h}}\left(\mathbb{P}_{h}^{2, \star} q(x), \mathbb{P}_{h}^{1, \star} p(x) ; \bar{\varphi}_{h}\right)_{j}=\widetilde{P_{h}}\left(q(x), p(x) ; \bar{\varphi}_{h}\right)_{j} \quad \forall \bar{\varphi}_{h} \in P^{k}\left(I_{j}\right),
\end{aligned}
$$

where $\widetilde{P_{h}}$ is given as

$$
\begin{aligned}
\widetilde{P_{h}}\left(p(x), q(x) ; \varphi_{h}\right)_{j}= & \frac{1}{\tau_{\max }}\left(\int_{x_{j-\frac{1}{2}}}^{x_{j}} p(x+h / 2) \varphi_{h}(x) \mathrm{d} x+\int_{x_{j}}^{x_{j+\frac{1}{2}}} p(x-h / 2) \varphi_{h}(x) \mathrm{d} x\right. \\
& \left.-\int_{x_{j-\frac{1}{2}}}^{x_{j+\frac{1}{2}}} p(x) \varphi_{h}(x) \mathrm{d} x\right)+\int_{x_{j-\frac{1}{2}}}^{x_{j}} q(x+h / 2)\left(\varphi_{h}(x)\right)_{x} \mathrm{~d} x \\
& +\int_{x_{j}}^{x_{j+\frac{1}{2}}} q(x-h / 2)\left(\varphi_{h}(x)\right)_{x} \mathrm{~d} x-q\left(x_{j}\right)\left(\varphi_{h}\left(x_{j+\frac{1}{2}}^{-}\right)-\varphi_{h}\left(x_{j-\frac{1}{2}}^{+}\right)\right),
\end{aligned}
$$

Similarly, we can define $\mathbb{Q}_{h}^{\star}(p, q):=\left(\mathbb{Q}_{h}^{1, \star} p, \mathbb{Q}_{h}^{2, \star} q\right) \in\left[W_{h}\right]^{2}$ such that

$$
\begin{aligned}
& \int_{I_{j+\frac{1}{2}}} \mathbb{Q}_{h}^{1, \star} p(x) \mathrm{d} x=\int_{I_{j+\frac{1}{2}}} p(x) \mathrm{d} x, \\
& \int_{I_{j+\frac{1}{2}}} \mathbb{Q}_{h}^{2, \star} q(x) \mathrm{d} x=\int_{I_{j+\frac{1}{2}}} q(x) \mathrm{d} x, \\
& \widetilde{Q_{h}}\left(\mathbb{Q}_{h}^{1, \star} p(x), \mathbb{Q}_{h}^{2, \star} q(x) ; \psi_{h}\right)_{j+\frac{1}{2}}=\widetilde{Q}_{h}\left(p(x), q(x) ; \psi_{h}\right)_{j+\frac{1}{2}}, \quad \forall \psi_{h} \in P^{k}\left(I_{j+\frac{1}{2}}\right), \\
& \widetilde{Q_{h}}\left(\mathbb{Q}_{h}^{2, \star} q(x), \mathbb{Q}_{h}^{1, \star} p(x) ; \bar{\psi}_{h}\right)_{j+\frac{1}{2}}=\widetilde{Q}_{h}\left(q(x), p(x) ; \bar{\psi}_{h}\right)_{j+\frac{1}{2}}, \quad \forall \bar{\psi}_{h} \in P^{k}\left(I_{j+\frac{1}{2}}\right),
\end{aligned}
$$


where $\widetilde{Q_{h}}$ is given as

$$
\begin{aligned}
\widetilde{Q_{h}} & \left.p(x), q(x) ; \varphi_{h}\right)_{j+\frac{1}{2}} \\
= & \frac{1}{\tau_{\max }}\left(\int_{x_{j}}^{x_{j+\frac{1}{2}}} p(x+h / 2) \psi_{h}(x) \mathrm{d} x+\int_{x_{j+\frac{1}{2}}}^{x_{j+1}} p(x-h / 2) \psi_{h}(x) \mathrm{d} x\right. \\
& \left.-\int_{x_{j}}^{x_{j+1}} p(x) \psi_{h}(x) \mathrm{d} x\right)+\int_{x_{j}}^{x_{j+\frac{1}{2}}} q(x+h / 2)\left(\psi_{h}(x)\right)_{x} \mathrm{~d} x \\
& +\int_{x_{j+\frac{1}{2}}}^{x_{j+1}} q(x-h / 2)\left(\psi_{h}(x)\right)_{x} \mathrm{~d} x-q\left(x_{j+\frac{1}{2}}\right)\left(\psi_{h}\left(x_{j+1}^{-}\right)-\psi_{h}\left(x_{j}^{+}\right)\right) .
\end{aligned}
$$

Next, we prove the projections $\mathbb{P}_{h}^{\star}$ and $\mathbb{Q}_{h}^{\star}$ are well defined. Note that the projections are local, so we only consider the projections defined on the reference interval $[-1,1]$. In this case, we have $h=2, \tau_{\max }=2 \alpha$. The projection can be viewed as the extension of the special projection (2.12) in [22]. Our coupled projections are applied to both functions by using the shifting technique. Without loss of generality, we only consider $\mathbb{P}_{h}^{\star}$ and the analysis of $\mathbb{Q}_{h}^{\star}$ is similar.

Lemma 3.2. The projection $\mathbb{P}_{h}^{\star}$ defined by (3.10a)-(3.10d) on the interval $[-1,1]$ exists and is unique for any functions $p, q \in H^{1}([-1,1])$. In particular, the projection is bounded in the $L^{\infty}$ norm, i.e.

$$
\left\|\mathbb{P}_{h}^{1, \star} p\right\|_{\infty}+\left\|\mathbb{P}_{h}^{2, \star} q\right\|_{\infty} \leq C(k)\left(\|p\|_{\infty}+\|q\|_{\infty}\right),
$$

where $C(k)$ is a constant that only depends on $k$ but is independent of $p, q$.

Proof. We provide the proof of this lemma in the Appendix A.1.

Since the projections $\mathbb{P}_{h}^{\star}$ and $\mathbb{Q}_{h}^{\star}$ are $k$-th degree polynomial preserving local projections, the standard approximation theory, Theorem 3.1.5 in [5] applies, i.e. for smooth functions $p, q$,

$$
\begin{aligned}
&\left\|\mathbb{P}_{h}^{1, \star} p-p\right\|+\left\|\mathbb{P}_{h}^{2, \star} q-q\right\|+h^{\frac{1}{2}}\left\|\mathbb{P}_{h}^{1, \star} p-p\right\|_{\Gamma_{h}}+h^{\frac{1}{2}}\left\|\mathbb{P}_{h}^{2, \star} q-q\right\|_{\Gamma_{h}} \lesssim h^{k+1}, \\
&\left\|\mathbb{Q}_{h}^{1, \star} p-p\right\|+\left\|\mathbb{Q}_{h}^{2, \star} q-q\right\|+h^{\frac{1}{2}}\left\|\mathbb{Q}_{h}^{1, \star \star} p-p\right\|_{\Gamma_{h}}+h^{\frac{1}{2}}\left\|\mathbb{Q}_{h}^{2, \star} q-q\right\|_{\Gamma_{h}} \lesssim h^{k+1} .
\end{aligned}
$$

When $p, q$ both belong to $P^{k+1}$, we have the following lemma.

Lemma 3.3. Assume that $p(x)=a x^{k+1}$ and $q(x)=b x^{k+1}$, where $a, b$ are constants. Then we have the projections $\mathbb{P}_{h}^{\star}(p, q)=\left(\mathbb{P}_{h}^{1, \star} p, \mathbb{P}_{h}^{2, \star} q\right)$ and $\mathbb{Q}_{h}^{\star}(p, q)=\left(\mathbb{Q}_{h}^{1, \star} p, \mathbb{Q}_{h}^{2, \star} q\right)$. Therefore $\forall x \in\left[x_{j-\frac{1}{2}}, x_{j+\frac{1}{2}}\right]$ we have

$$
\left(a x^{k+1}-\mathbb{P}_{h}^{1, \star} p(x), b x^{k+1}-\mathbb{P}_{h}^{2, \star} q(x)\right)=\left(a(x \mp h / 2)^{k+1}-\mathbb{Q}_{h}^{1, \star} p(x \mp h / 2), b(x \mp h / 2)^{k+1}-\mathbb{Q}_{h}^{2, \star} q(x \mp h / 2)\right) .
$$

Proof. The proof of this lemma is provided in the Appendix A.2.

Besides the standard approximation results (3.15) and (3.16), the special projections $\mathbb{P}_{h}^{\star}$ and $\mathbb{Q}_{h}^{\star}$ also have the following superconvergence result.

Proposition 3.4. Assume that $p, q$ are both $(k+1)$-th degree polynomial functions in $P^{k+1}\left(K_{j}\right)$, $K_{j}=\left[x_{j-1}, x_{j+\frac{3}{2}}\right]$. For a uniform partition on $\Omega$, set $p_{I}=\mathbb{P}_{h}^{1, \star \star} p \in V_{h}, q_{I}=\mathbb{P}_{h}^{2, \star} q \in V_{h}$ and $r_{I}=\mathbb{Q}_{h}^{1, \star} p \in W_{h}$, $s_{I}=\mathbb{Q}_{h}^{2, \star} q \in W_{h}$. Then we have

$$
\begin{array}{ll}
B_{1}\left(r_{I}, p_{I}, s_{I} ; \varphi_{h}\right)_{j}=B_{1}\left(p, p, q ; \varphi_{h}\right)_{j}, & \forall \varphi_{h} \in P^{k}\left(I_{j}\right), \\
B_{1}\left(s_{I}, q_{I}, r_{I} ; \bar{\varphi}_{h}\right)_{j}=B_{1}\left(q, q, p ; \bar{\varphi}_{h}\right)_{j}, & \forall \bar{\varphi}_{h} \in P^{k}\left(I_{j}\right), \\
B_{2}\left(p_{I}, r_{I}, q_{I} ; \psi_{h}\right)_{j+\frac{1}{2}}=B_{2}\left(p, p, q ; \psi_{h}\right)_{j+\frac{1}{2}}, & \forall \psi_{h} \in P^{k}\left(I_{j+\frac{1}{2}}\right), \\
B_{2}\left(q_{I}, s_{I}, p_{I} ; \bar{\psi}_{h}\right)_{j+\frac{1}{2}}=B_{2}\left(q, q, p ; \bar{\psi}_{h}\right)_{j+\frac{1}{2}}, & \forall \bar{\psi}_{h} \in P^{k}\left(I_{j+\frac{1}{2}}\right) .
\end{array}
$$


Proof. We give the proof of this proposition in the Appendix A.3.

Next we prove the optimal error estimates for the central DG scheme (3.2) on the uniform overlapping Cartesian meshes, stated in the following theorem.

Theorem 3.5 (Error estimates). Suppose that $p, q \in H^{k+2}(\Omega)$ are the exact solutions of the equation (3.1) with smooth initial condition $p(\cdot, 0), q(\cdot, 0) \in H^{k+2}(\Omega)$. The numerical solution $p_{h}, q_{h}, r_{h}, s_{h}$ of the central $D G$ scheme (3.2) satisfies the following $L^{2}$ error estimate

$$
\left\|p-p_{h}\right\|^{2}+\left\|p-r_{h}\right\|^{2}+\left\|q-q_{h}\right\|^{2}+\left\|q-s_{h}\right\|^{2} \lesssim h^{2 k+2} .
$$

Proof. In the error equation (3.8), we take

$$
\begin{array}{ll}
\varphi_{h}=p_{h}-\mathbb{P}_{h}^{1, \star \star} p, \quad \bar{\varphi}_{h}=q_{h}-\mathbb{P}_{h}^{2, \star} q, \\
\psi_{h}=r_{h}-\mathbb{Q}_{h}^{1, \star} p, \quad \bar{\psi}_{h}=s_{h}-\mathbb{Q}_{h}^{2, \star} q,
\end{array}
$$

and define

Then we obtain

$$
\begin{array}{ll}
p_{e}=p-\mathbb{P}_{h}^{1, \star} p, & q_{e}=q-\mathbb{P}_{h}^{2, \star} q, \\
r_{e}=p-\mathbb{Q}_{h}^{1, \star} p, & s_{e}=q-\mathbb{Q}_{h}^{2, \star} q .
\end{array}
$$

$$
A_{j}\left(\varphi_{h}, \psi_{h}, \bar{\varphi}_{h}, \bar{\psi}_{h} ; \varphi_{h}, \psi_{h}, \bar{\varphi}_{h}, \bar{\psi}_{h}\right)=A_{j}\left(p_{e}, r_{e}, q_{e}, s_{e} ; \varphi_{h}, \psi_{h}, \bar{\varphi}_{h}, \bar{\psi}_{h}\right) .
$$

For the left-hand side of (3.25), we mimic the proof of the $L^{2}$ stability to conclude

$$
\sum_{j} A_{j}\left(\varphi_{h}, \psi_{h}, \bar{\varphi}_{h}, \bar{\psi}_{h} ; \varphi_{h}, \psi_{h}, \bar{\varphi}_{h}, \bar{\psi}_{h}\right)=\frac{1}{2} \frac{d}{d t} \int_{0}^{1} \varphi_{h}^{2}+\psi_{h}^{2}+\bar{\varphi}_{h}^{2}+\bar{\psi}_{h}^{2} \mathrm{~d} x+\frac{1}{\tau_{\max }} \int_{0}^{1}\left(\varphi_{h}-\psi_{h}\right)^{2}+\left(\bar{\varphi}_{h}-\bar{\psi}_{h}\right)^{2} \mathrm{~d} x
$$

For the right-hand side of (3.25), we rewrite it as a sum of five terms

$$
A_{j}\left(p_{e}, r_{e}, q_{e}, s_{e} ; \varphi_{h}, \psi_{h}, \bar{\varphi}_{h}, \bar{\psi}_{h}\right)=\sum_{\ell=1}^{5} A_{j}^{\ell},
$$

where $A_{j}^{1}, \ldots, A_{j}^{5}$ are given as

$$
\begin{aligned}
& A_{j}^{1}=\int_{I_{j}}\left(p_{e}\right)_{t} \varphi_{h}+\left(q_{e}\right)_{t} \bar{\varphi}_{h} \mathrm{~d} x+\int_{I_{j+\frac{1}{2}}}\left(r_{e}\right)_{t} \psi_{h}+\left(s_{e}\right)_{t} \bar{\psi}_{h} \mathrm{~d} x, \\
& A_{j}^{2}=-B_{1}\left(r_{e}, p_{e}, s_{e} ; \varphi_{h}\right)_{j}, \quad A_{j}^{3}=-B_{1}\left(s_{e}, q_{e}, r_{e} ; \bar{\varphi}_{h}\right)_{j}, \\
& A_{j}^{4}=-B_{2}\left(p_{e}, r_{e}, q_{e} ; \psi_{h}\right)_{j+\frac{1}{2}}, \quad A_{j}^{5}=-B_{2}\left(q_{e}, s_{e}, p_{e} ; \bar{\psi}_{h}\right)_{j+\frac{1}{2}},
\end{aligned}
$$

and we next estimate each term separately. For $A_{j}^{1}$, by using the Cauchy-Schwarz inequality and the optimal estimates of the projection error (3.15) and (3.16), we have

$$
\sum_{j} A_{j}^{1} \lesssim h^{k+1}\left(\left\|\varphi_{h}\right\|^{2}+\left\|\bar{\varphi}_{h}\right\|^{2}+\left\|\psi_{h}\right\|^{2}+\left\|\bar{\psi}_{h}\right\|^{2}\right)^{\frac{1}{2}} .
$$

For $A_{j}^{\ell}, \ell=2, \ldots, 5$, we use Proposition 3.4 to obtain

$$
\begin{aligned}
B_{1}\left(r_{e}, p_{e}, s_{e} ; \varphi_{h}\right)_{j}= & B_{1}\left(p-\mathbb{Q}_{h}^{1, \star} p, p-\mathbb{P}_{h}^{1, \star} p, q-\mathbb{Q}_{h}^{2, \star} q ; \varphi_{h}\right)_{j} \\
& -B_{1}\left(T p-\mathbb{Q}_{h}^{1, \star} T p, T p-\mathbb{P}_{h}^{1, \star} T p, T q-\mathbb{Q}_{h}^{2, \star} T q ; \varphi_{h}\right)_{j} \\
= & B_{1}\left(\Pi p-\mathbb{Q}_{h}^{1, \star} \Pi p, \Pi p-\mathbb{P}_{h}^{1, \star} \Pi p, \Pi q-\mathbb{Q}_{h}^{2, \star} \Pi q ; \varphi_{h}\right)_{j},
\end{aligned}
$$


where $\Pi p=p-T p, \Pi q=q-T q$, for any $T p, T q \in P^{k+1}\left(K_{j}\right)$. Therefore, by using the Cauchy-Schwarz inequality, the special projection property (3.14), the Bramble-Hilbert lemma, Theorem 4.1.3 in [5], and the inverse inequality in (3.9) for $\varphi_{h}$, we have

$$
\sum_{j}\left|A_{j}^{2}\right| \lesssim h^{k+1}\left\|\varphi_{h}\right\|
$$

Similarly, we also have the estimates for $A_{j}^{3}, A_{j}^{4}, A_{j}^{5}$.

$$
\sum_{j}\left|A_{j}^{3}\right| \lesssim h^{k+1}\left\|\bar{\varphi}_{h}\right\|, \quad \sum_{j}\left|A_{j}^{4}\right| \lesssim h^{k+1}\left\|\psi_{h}\right\|, \quad \sum_{j}\left|A_{j}^{5}\right| \lesssim h^{k+1}\left\|\bar{\psi}_{h}\right\| .
$$

Then we substitute the estimates (3.29), (3.31) and (3.32) into (3.26), and obtain

$$
\frac{1}{2} \frac{\mathrm{d}}{\mathrm{d} t} \int_{0}^{1} \varphi_{h}^{2}+\psi_{h}^{2}+\bar{\varphi}_{h}^{2}+\bar{\psi}_{h}^{2} \mathrm{~d} x \lesssim h^{k+1}\left(\left\|\varphi_{h}\right\|^{2}+\left\|\bar{\varphi}_{h}\right\|^{2}+\left\|\psi_{h}\right\|^{2}+\left\|\bar{\psi}_{h}\right\|^{2}\right)^{\frac{1}{2}} .
$$

Together with the projection error (3.15), (3.16) and the initial error estimates (2.5), we finally obtain the desired error estimate (3.22).

\subsubsection{Dispersion analysis}

In this subsection, we derive the dispersion error for the central DG scheme (3.2) with $P^{0}, P^{1}$ and $P^{2}$ elements. The technique of dispersion analysis comes from [3]. As usually required in the dispersion analysis, we use a uniform mesh, i.e. $h_{j}=h_{j+\frac{1}{2}}=h, \forall j$. We assume that the initial condition has the form

$$
p_{0}(x)=p_{0} e^{i k x}, \quad q_{0}(x)=q_{0} e^{i k x},
$$

then the exact solution is given by

$$
\begin{aligned}
& p(x, t)=\frac{p_{0}+q_{0}}{2} e^{i k(x-t)}+\frac{p_{0}-q_{0}}{2} e^{i k(x+t)}, \\
& q(x, t)=\frac{p_{0}+q_{0}}{2} e^{i k(x-t)}-\frac{p_{0}-q_{0}}{2} e^{i k(x+t)} .
\end{aligned}
$$

Clearly, the exact solution is composed of two waves $e^{i(k x+\omega t)}$ with the dispersion relation $\omega= \pm k$.

$\boldsymbol{P}^{\mathbf{0}}$ polynomials. For the case of piecewise constant polynomial space, we assume $\left.p_{h}\right|_{I_{j}}=p_{j},\left.q_{h}\right|_{I_{j}}=q_{j}$, $\left.r_{h}\right|_{I_{j+\frac{1}{2}}}=r_{j}$ and $\left.s_{h}\right|_{I_{j+\frac{1}{2}}}=s_{j}$. From (3.2), we can obtain the following relation

$$
\left(\begin{array}{c}
p_{j} \\
q_{j} \\
r_{j} \\
s_{j}
\end{array}\right)_{t}=A_{1}\left(\begin{array}{c}
p_{j-1} \\
q_{j-1} \\
r_{j-1} \\
s_{j-1}
\end{array}\right)+A_{2}\left(\begin{array}{c}
p_{j} \\
q_{j} \\
r_{j} \\
s_{j}
\end{array}\right)+A_{3}\left(\begin{array}{c}
p_{j+1} \\
q_{j+1} \\
r_{j+1} \\
s_{j+1}
\end{array}\right)
$$

where $A_{1}, A_{2}, A_{3}$ are $4 \times 4$ matrices. From the assumption that the wave takes the form $p_{j}(t)=\hat{p}(t) e^{i k x_{j}}$, $q_{j}(t)=\hat{q}(t) e^{i k x_{j}}, r_{j}(t)=\hat{r}(t) e^{i k x_{j+\frac{1}{2}}}, s_{j}(t)=\hat{s}(t) e^{i k x_{j+\frac{1}{2}}}$, then the above relation can be written as

$$
\hat{\boldsymbol{U}}_{t}=G \hat{\boldsymbol{U}}, \quad \hat{\boldsymbol{U}}=(\hat{p}, \hat{q}, \hat{r}, \hat{s})^{T},
$$

where $G$ is the amplification matrix given by

$$
G=\frac{1}{\alpha h}\left(\begin{array}{cc}
-I_{2} & A \\
A & -I_{2}
\end{array}\right), \quad A=\left(\begin{array}{cc}
\cos (w) & -2 i \alpha \sin (w) \\
-2 i \alpha \sin (w) & \cos (w)
\end{array}\right), w=\frac{k h}{2},
$$


and $I_{2}$ is the $2 \times 2$ identity matrix. The matrix $G$ has four eigenvalues $\lambda_{1,2}, \lambda_{3,4}$, and through calculation we obtain

$$
\begin{aligned}
& \tilde{\omega}_{1,2}=\frac{\lambda_{1,2}}{i}= \pm k+i \frac{k^{2} h}{8 \alpha} \mp \frac{k^{3} h^{2}}{24}-i \frac{k^{4} h^{3}}{384 \alpha}+O\left(k(k h)^{4}\right), \\
& \tilde{\omega}_{3,4}=\frac{\lambda_{3,4}}{i}=i \frac{2}{\alpha h} \mp k-i \frac{k^{2} h}{8 \alpha} \pm \frac{k^{3} h^{2}}{24}+i \frac{k^{4} h^{3}}{384 \alpha}+O\left(k(k h)^{4}\right) .
\end{aligned}
$$

Clearly, $\tilde{\omega}_{1,2}$ correspond to the physical modes, and $\tilde{\omega}_{3,4}$ are the spurious modes. The leading errors in the physical modes are a first order dissipation error and a second order dispersion error. The spurious modes, on the other hand, get damped exponentially fast in time, due to the leading imaginary part of $\tilde{\omega}_{3,4}$ being positive and proportional to $O\left(h^{-1}\right)$.

$\boldsymbol{P}^{\mathbf{1}}$ polynomials. The analysis for the $P^{1}$ element space is similar to that for the $P^{0}$ elements. By choosing the basis functions on each element $I_{j}$ to be $\varphi_{1}=-\xi+1 / 2, \varphi_{2}=\xi+1 / 2$, with $\xi=\left(x-x_{j}\right) / h$, and $I_{j+\frac{1}{2}}$ to be $\psi_{1}=-\eta+1 / 2, \psi_{2}=\eta+1 / 2$ with $\eta=\left(x-x_{j+\frac{1}{2}}\right) / h$. Then the numerical solution on $I_{j}$ can be written as $p_{h}=p_{j}^{1} \varphi_{1}+p_{j}^{2} \varphi_{2}, q_{h}=q_{j}^{1} \varphi_{1}+q_{j}^{2} \varphi_{2}$ and on $I_{j+\frac{1}{2}}$ can be written as $r_{h}=r_{j}^{1} \psi_{1}+r_{j}^{2} \psi_{2}, s_{h}=s_{j}^{1} \psi_{1}+s_{j}^{2} \psi_{2}$. Similar as before, we obtain the following ODE

$$
\hat{\boldsymbol{U}}_{t}=G \hat{\boldsymbol{U}}, \quad \hat{\boldsymbol{U}}=\left(\hat{p}^{1}, \hat{p}^{2}, \hat{q}^{1}, \hat{q}^{2}, \hat{r}^{1}, \hat{r}^{2}, \hat{s}^{1}, \hat{s}^{2}\right)^{T},
$$

in which the amplification matrix $G$ is given by

$$
G=\frac{1}{\alpha h}\left(\begin{array}{cc}
-I_{4} & A \\
A & -I_{4}
\end{array}\right), \quad A=\left(\begin{array}{ll}
A_{1} & A_{2} \\
A_{2} & A_{1}
\end{array}\right)
$$

where $I_{4}$ is the $4 \times 4$ identity matrix and $A_{1}$ and $A_{2}$ are defined as

$$
A_{1}=\frac{1}{8}\left(\begin{array}{cc}
3 e^{-i w}-e^{i w} & 7 e^{-i w}-e^{i w} \\
-e^{-i w}+7 e^{i w}-e^{-i w}+3 e^{i w}
\end{array}\right), \quad A_{2}=\frac{\alpha}{4}\left(e^{-i w}-e^{i w}\right)\left(\begin{array}{cc}
5 & -1 \\
-1 & 5
\end{array}\right), w=\frac{k h}{2} .
$$

The matrix $G$ has eight distinct eigenvalues $\lambda_{i}, i=1, \ldots, 8$, and we obtain

$$
\begin{aligned}
& \hat{\omega}_{1,2}=\frac{\lambda_{1,2}}{i}= \pm k+i \frac{h^{3} k^{4}\left(3+16 \alpha^{2}\right)}{1152 \alpha} \mp \frac{h^{4} k^{5}\left(81+80 \alpha^{2}\right)}{17280}+O\left(k(k h)^{5}\right), \\
& \hat{\omega}_{3,4}=i \frac{1}{2 h}+O(1), \quad \hat{\omega}_{5,6}=i \frac{3}{2 h}+O(1), \quad \hat{\omega}_{7,8}=i \frac{2}{h}+O(1) .
\end{aligned}
$$

The leading errors in the physical modes $\hat{\omega}_{1,2}$ are a third order dissipation error and a fourth order dispersion error. Clearly we can see the magnitude of the leading error term $i \frac{h^{3} k^{4}\left(3+16 \alpha^{2}\right)}{1152 \alpha}$ in (3.36) reaches its minimum at $\alpha=\sqrt{3} / 4$. The spurious modes, on the other hand, get damped exponentially fast in time, due to the leading imaginary parts of $\hat{\omega}_{3,4,5,6,7,8}$ being positive and proportional $O\left(h^{-1}\right)$.

$P^{2}$ polynomials. For the piecewise quadratic polynomial case, we choose the basis functions on each element $I_{j}$ to be $\varphi_{1}=2 \xi(\xi-1 / 2), \varphi_{2}=-4\left(\xi^{2}-1 / 4\right), \varphi_{3}=2 \xi(\xi+1 / 2)$ where $\xi=\left(x-x_{j}\right) / h$ and on each element $I_{j+\frac{1}{2}}$ to be $\psi_{1}=2 \eta(\eta-1 / 2), \psi_{2}=-4\left(\eta^{2}-1 / 4\right), \psi_{3}=2 \eta(\eta+1 / 2)$ where $\eta=\left(x-x_{j+\frac{1}{2}}\right) / h$. Similar derivations show that there are twelve eigenvalues of the amplification matrix, and

$$
\begin{aligned}
\hat{\omega}_{1,2} & =\frac{\lambda_{1,2}}{i}= \pm k+i \frac{53 k^{6} h^{5}}{2918400} \mp \frac{24089 k^{7} h^{6}}{646912000}+O\left(k(k h)^{8}\right) \text { for } \alpha=1, \\
\hat{\omega}_{3,4} & =i \frac{2}{\alpha} \frac{1}{h}+O(1), \quad \hat{\omega}_{5,6,7,8}=i \frac{27 \alpha \pm \sqrt{9 \alpha^{2}-3840 \alpha^{4}}}{16 \alpha^{2}} \frac{1}{h}+O(1), \\
\hat{\omega}_{9,10,11,12} & =i \frac{5 \alpha \pm \sqrt{9 \alpha^{2}-3840 \alpha^{4}}}{16 \alpha^{2}} \frac{1}{h}+O(1) .
\end{aligned}
$$


The leading errors in the physical modes $\hat{\omega}_{1,2}$ are a fifth order dissipation error and a sixth order dispersion error. Since the formulation of the coefficient of the leading term is too long to simplify, here, we just give the case for $\alpha=1$. For the spurious modes, we can verify by basic algebraic manipulations that the imaginary part of the leading term of $\hat{\omega}_{3, \ldots, 12}$ is positive and propositional $O\left(h^{-1}\right)$ for arbitrary $\alpha>0$. Thus they can get damped exponentially fast in time.

Remark 3.6. Compared with [3], we obtain the same dissipation error order and dispersion error order. Besides, we provide the best parameter $\alpha$ for $P^{1}$ element to reach the minimum dissipation error. For $P^{0}$ and $P^{2}$ elements, the dissipation error is decreasing as $\alpha$ increases. For the dissipation and dispersion analysis of general $P^{k}$, it will be left to our future work.

\subsection{Analysis of the central DG method in multidimensions}

For $d=2$ in (1.3), we have the following two dimensional problem

$$
\begin{cases}p_{t}=c q_{x}+c r_{y}, & (x, y) \in \Omega, t>0, \\ q_{t}=c p_{x}, & (x, y) \in \Omega, t>0, \\ r_{t}=c p_{y}, & (x, y) \in \Omega, t>0, \\ p(x, y, 0)=p_{0}(x, y), & q(x, y, 0)=q_{0}(x, y), \quad r(x, y, 0)=r_{0}(x, y),\end{cases}
$$

again with periodic boundary conditions. We assume $\Omega=[0,1]^{2}$ and $c=-1$.

Let $\left\{K_{i, j}=\left[x_{i-\frac{1}{2}}, x_{i+\frac{1}{2}}\right] \times\left[y_{j-\frac{1}{2}}, y_{j+\frac{1}{2}}\right]\right\}, i=1, \ldots, N_{x}, j=1, \ldots, N_{y}$ be a partition of $\Omega$ into rectangular cells. Let $\left\{K_{i+\frac{1}{2}, j+\frac{1}{2}}=\left[x_{i}, x_{i+1}\right] \times\left[y_{j}, y_{j+1}\right]\right\}, i=1, \ldots, N_{x}, j=1, \ldots, N_{y}$ be a dual mesh with $x_{i}=\left(x_{i-\frac{1}{2}}+x_{i+\frac{1}{2}}\right) / 2$ and $y_{j}=\left(y_{j-\frac{1}{2}}+y_{j+\frac{1}{2}}\right) / 2$. Then we have the finite element spaces as follows.

$$
\begin{aligned}
V_{h} & :=\left\{v \in L^{2}(\Omega):\left.\varphi\right|_{K_{i, j}} \in P^{k}\left(K_{i, j}\right), \forall i, j\right\}, \\
W_{h} & :=\left\{v \in L^{2}(\Omega):\left.\bar{\varphi}\right|_{K_{i+\frac{1}{2}, j+\frac{1}{2}}} \in P^{k}\left(K_{i+\frac{1}{2}, j+\frac{1}{2}}\right), \forall i, j\right\},
\end{aligned}
$$

where $P^{k}\left(K_{i, j}\right)$ denotes the space of polynomials of degrees at most $k$ defined on $K_{i, j}$; no continuity is assumed across cell boundaries, and $P^{k}\left(K_{i+\frac{1}{2}, j+\frac{1}{2}}\right)$ denotes the space of polynomials of degrees at most $k$ defined on $K_{i+\frac{1}{2}, j+\frac{1}{2}}$; no continuity is assumed across cell boundaries. We denote

$$
\begin{aligned}
h_{x}^{i} & =x_{i+\frac{1}{2}}-x_{i-\frac{1}{2}}, \quad h_{y}^{j}=y_{j+\frac{1}{2}}-y_{j-\frac{1}{2}}, \\
h_{x}^{i+\frac{1}{2}} & =x_{i+1}-x_{i}, \quad h_{y}^{j+\frac{1}{2}}=y_{j+1}-y_{j}, \\
h & =\max \left(h_{x}^{i}, h_{y}^{j}, h_{x}^{i+\frac{1}{2}}, h_{y}^{j+\frac{1}{2}}\right) .
\end{aligned}
$$

The semidiscrete central DG scheme for solving equation (3.37) is defined as follows. Find the numerical solutions $p_{h}, q_{h}, r_{h} \in V_{h}$ and $u_{h}, v_{h}, w_{h} \in W_{h}$ such that for all test functions $\varphi_{h}, \bar{\varphi}_{h}, \tilde{\varphi}_{h} \in V_{h}$ and $\psi_{h}, \bar{\psi}_{h}, \tilde{\psi}_{h} \in W_{h}$ and all $i, j$, we have

$$
\begin{aligned}
\int_{K_{i, j}}\left(p_{h}\right)_{t} \varphi_{h} \mathrm{~d} x \mathrm{~d} y & =\mathcal{B}_{1}\left(u_{h}, p_{h}, v_{h}, w_{h} ; \varphi_{h}\right)_{i, j}, \\
\int_{K_{i, j}}\left(q_{h}\right)_{t} \bar{\varphi}_{h} \mathrm{~d} x \mathrm{~d} y & =\mathcal{B}_{2}\left(v_{h}, q_{h}, u_{h} ; \bar{\varphi}_{h}\right)_{i, j}, \\
\int_{K_{i, j}}\left(r_{h}\right)_{t} \tilde{\varphi}_{h} \mathrm{~d} x \mathrm{~d} y & =\mathcal{B}_{3}\left(w_{h}, r_{h}, u_{h} ; \tilde{\varphi}_{h}\right)_{i, j}, \\
\int_{K_{i+\frac{1}{2}, j+\frac{1}{2}}}\left(u_{h}\right)_{t} \psi_{h} \mathrm{~d} x \mathrm{~d} y & =\mathcal{B}_{1}\left(p_{h}, u_{h}, q_{h}, r_{h} ; \psi_{h}\right)_{i+\frac{1}{2}, j+\frac{1}{2}},
\end{aligned}
$$




$$
\begin{aligned}
\int_{K_{i+\frac{1}{2}, j+\frac{1}{2}}}\left(v_{h}\right)_{t} \bar{\psi}_{h} \mathrm{~d} x \mathrm{~d} y & =\mathcal{B}_{2}\left(q_{h}, v_{h}, p_{h} ; \bar{\psi}_{h}\right)_{i+\frac{1}{2}, j+\frac{1}{2}}, \\
\int_{K_{i+\frac{1}{2}, j+\frac{1}{2}}}\left(w_{h}\right)_{t} \tilde{\psi}_{h} \mathrm{~d} x \mathrm{~d} y & =\mathcal{B}_{3}\left(r_{h}, w_{h}, p_{h} ; \tilde{\psi}_{h}\right)_{i+\frac{1}{2}, j+\frac{1}{2}},
\end{aligned}
$$

where $\mathcal{B}_{1}, \mathcal{B}_{2}, \mathcal{B}_{3}$ are given as

$$
\begin{aligned}
\mathcal{B}_{1}\left(u_{h}, p_{h}, v_{h}, w_{h} ; \varphi_{h}\right)_{i, j}= & \frac{1}{\tau_{\max }} \int_{K_{i, j}}\left(u_{h}-p_{h}\right) \varphi_{h} \mathrm{~d} x \mathrm{~d} y+\int_{K_{i, j}}\left(v_{h}\left(\varphi_{h}\right)_{x}+w_{h}\left(\varphi_{h}\right)_{y}\right) \mathrm{d} x \mathrm{~d} y \\
& -\int_{y_{j-\frac{1}{2}}}^{y_{j+\frac{1}{2}}} v_{h}\left(x_{i+\frac{1}{2}}, y\right) \varphi_{h}\left(x_{i+\frac{1}{2}}^{-}, y\right)-v_{h}\left(x_{i-\frac{1}{2}}, y\right) \varphi_{h}\left(x_{i-\frac{1}{2}}^{+}, y\right) \mathrm{d} y \\
& -\int_{x_{i-\frac{1}{2}}}^{x_{i+\frac{1}{2}}} w_{h}\left(x, y_{j+\frac{1}{2}}\right) \varphi_{h}\left(x, y_{j+\frac{1}{2}}^{-}\right)-w_{h}\left(x, y_{j-\frac{1}{2}}\right) \varphi_{h}\left(x, y_{j-\frac{1}{2}}^{+}\right) \mathrm{d} x, \\
\mathcal{B}_{2}\left(v_{h}, q_{h}, u_{h} ; \bar{\varphi}_{h}\right)_{i, j}= & \frac{1}{\tau_{\max }} \int_{K_{i, j}}\left(v_{h}-q_{h}\right) \bar{\varphi}_{h} \mathrm{~d} x+\int_{K_{i, j}} u_{h}\left(\bar{\varphi}_{h}\right)_{x} \mathrm{~d} x \mathrm{~d} y \\
& -\int_{y_{j-\frac{1}{2}}}^{y_{j+\frac{1}{2}}} u_{h}\left(x_{i+\frac{1}{2}}, y\right) \bar{\varphi}_{h}\left(x_{i+\frac{1}{2}}^{-}, y\right)-u_{h}\left(x_{i-\frac{1}{2}}, y\right) \bar{\varphi}_{h}\left(x_{i-\frac{1}{2}}^{+}, y\right) \mathrm{d} y, \\
\mathcal{B}_{3}\left(w_{h}, r_{h}, u_{h} ; \tilde{\varphi}_{h}\right)_{i, j}= & \frac{1}{\tau_{\max }} \int_{K_{i, j}}\left(w_{h}-r_{h}\right) \tilde{\varphi}_{h} \mathrm{~d} x+\int_{K_{i, j}} u_{h}\left(\tilde{\varphi}_{h}\right)_{y} \mathrm{~d} x \mathrm{~d} y \\
& -\int_{x_{i-\frac{1}{2}}}^{x_{i+\frac{1}{2}}} u_{h}\left(x, y_{j+\frac{1}{2}}\right) \tilde{\varphi}_{h}\left(x, y_{j+\frac{1}{2}}^{-}\right)-u_{h}\left(x, y_{j-\frac{1}{2}}\right) \tilde{\varphi}_{h}\left(x, y_{j-\frac{1}{2}}^{+}\right) \mathrm{d} y .
\end{aligned}
$$

In this subsection, we consider the semidiscrete central DG method for multidimensional problems. Without loss of generality, we derive the $L^{2}$ stability and prove the optimal a priori error estimates for the central DG scheme (3.38) in two dimensions $(d=2)$. We only consider the rectangular mesh and finite element space is piecewise $P^{k}$ element space. The analysis can be extended to the higher dimensional cases $d>2$ without any difficulties.

Theorem 3.7 ( $L^{2}$ stability). The approximations $p_{h}, q_{h}, r_{h} \in V_{h}$ and $u_{h}, v_{h}, w_{h} \in W_{h}$ of the semidiscrete central DG method (3.38) for the equation (3.37) has the following $L^{2}$ stability property

$$
\begin{aligned}
\frac{1}{2} \frac{\mathrm{d}}{\mathrm{d} t} & \left(\left\|p_{h}\right\|^{2}+\left\|q_{h}\right\|^{2}+\left\|r_{h}\right\|^{2}+\left\|u_{h}\right\|^{2}+\left\|v_{h}\right\|^{2}+\left\|w_{h}\right\|^{2}\right) \\
& =-\frac{1}{\tau_{\max }}\left(\int_{\Omega}\left(p_{h}-u_{h}\right)^{2} \mathrm{~d} x \mathrm{~d} y+\int_{\Omega}\left(q_{h}-v_{h}\right)^{2} \mathrm{~d} x \mathrm{~d} y+\int_{\Omega}\left(r_{h}-w_{h}\right)^{2} \mathrm{~d} x \mathrm{~d} y\right) \leq 0 .
\end{aligned}
$$

Proof. Taking $\left(\varphi_{h}, \bar{\varphi}_{h}, \tilde{\varphi}_{h}\right)=\left(p_{h}, q_{h}, r_{h}\right) \in\left[V_{h}\right]^{3}$ and $\left(\psi_{h}, \bar{\psi}_{h}, \tilde{\psi}_{h}\right)=\left(u_{h}, v_{h}, w_{h}\right) \in\left[W_{h}\right]^{3}$ in the scheme (3.38), summing up over $j$, and using the periodic boundary condition, we have

$$
\begin{aligned}
\frac{1}{2} \frac{\mathrm{d}}{\mathrm{d} t}\left(\left\|p_{h}\right\|^{2}+\left\|q_{h}\right\|^{2}+\left\|r_{h}\right\|^{2}+\left\|u_{h}\right\|^{2}+\left\|v_{h}\right\|^{2}+\left\|w_{h}\right\|^{2}\right) \\
=\sum_{i, j} \mathcal{B}_{1}\left(u_{h}, p_{h}, v_{h}, w_{h} ; p_{h}\right)_{i, j}+\mathcal{B}_{2}\left(v_{h}, q_{h}, u_{h} ; q_{h}\right)_{i, j}+\mathcal{B}_{3}\left(w_{h}, r_{h}, u_{h} ; r_{h}\right)_{i, j} \\
\quad+\sum_{i, j} \mathcal{B}_{1}\left(p_{h}, u_{h}, q_{h}, r_{h} ; u_{h}\right)_{i+\frac{1}{2}, j+\frac{1}{2}}+\mathcal{B}_{2}\left(q_{h}, v_{h}, p_{h} ; v_{h}\right)_{i+\frac{1}{2}, j+\frac{1}{2}} \\
\quad+\mathcal{B}_{3}\left(r_{h}, w_{h}, p_{h} ; w_{h}\right)_{i+\frac{1}{2}, j+\frac{1}{2}} \\
=-\frac{1}{\tau_{\max }} \int_{\Omega}\left(p_{h}-u_{h}\right)^{2}+\left(q_{h}-v_{h}\right)^{2}+\left(r_{h}-w_{h}\right)^{2} \mathrm{~d} x \mathrm{~d} y \leq 0 .
\end{aligned}
$$


Next, we start to derive the optimal error estimates for the central DG scheme (3.38). Assume the mesh is uniform, i.e. $h_{x}^{i}=h_{x}^{i+\frac{1}{2}}=h_{x}$ and $h_{y}^{j}=h_{y}^{j+\frac{1}{2}}=h_{y}$. We first define

$$
\begin{aligned}
& \mathcal{A}_{i, j}\left(p_{h}, q_{h}, r_{h}, u_{h}, v_{h}, w_{h} ; \varphi_{h}, \bar{\varphi}_{h}, \tilde{\varphi}_{h}, \psi_{h}, \bar{\psi}_{h}, \tilde{\psi}_{h}\right) \\
&=\int_{K_{i, j}} \partial_{t} p_{h} \varphi_{h} \mathrm{~d} x \mathrm{~d} y+\int_{K_{i, j}} \partial_{t} q_{h} \bar{\varphi}_{h} \mathrm{~d} x \mathrm{~d} y+\int_{K_{i, j}} \partial_{t} r_{h} \tilde{\varphi}_{h} \mathrm{~d} x \mathrm{~d} y \\
& \quad+\int_{K_{i+\frac{1}{2}, j+\frac{1}{2}}} \partial_{t} u_{h} \psi_{h} \mathrm{~d} x \mathrm{~d} y+\int_{K_{i+\frac{1}{2}, j+\frac{1}{2}}} \partial_{t} v_{h} \bar{\psi}_{h} \mathrm{~d} x \mathrm{~d} y+\int_{K_{i+\frac{1}{2}, j+\frac{1}{2}}} \partial_{t} w_{h} \tilde{\psi}_{h} \mathrm{~d} x \mathrm{~d} y \\
& \quad-\mathcal{B}_{1}\left(u_{h}, p_{h}, v_{h}, w_{h} ; \varphi_{h}\right)_{i, j}-\mathcal{B}_{2}\left(v_{h}, q_{h}, u_{h} ; \bar{\varphi}_{h}\right)_{i, j}-\mathcal{B}_{3}\left(w_{h}, r_{h}, u_{h} ; \tilde{\varphi}_{h}\right)_{i, j} \\
& \quad-\mathcal{B}_{1}\left(p_{h}, u_{h}, q_{h}, r_{h} ; \psi_{h}\right)_{i+\frac{1}{2}, j+\frac{1}{2}}-\mathcal{B}_{2}\left(q_{h}, v_{h}, p_{h} ; \bar{\psi}_{h}\right)_{i+\frac{1}{2}, j+\frac{1}{2}} \\
&-\mathcal{B}_{3}\left(r_{h}, w_{h}, p_{h} ; \tilde{\psi}_{h}\right)_{i+\frac{1}{2}, j+\frac{1}{2}}
\end{aligned}
$$

Clearly, $\forall i, j$ and $\forall\left(\varphi_{h}, \bar{\varphi}_{h}, \tilde{\varphi}_{h}\right) \in\left[V_{h}\right]^{3},\left(\psi_{h}, \bar{\psi}_{h}, \tilde{\psi}_{h}\right) \in\left[W_{h}\right]^{3}$ we have

$$
\mathcal{A}_{i, j}\left(p_{h}, q_{h}, r_{h}, u_{h}, v_{h}, w_{h} ; \varphi_{h}, \bar{\varphi}_{h}, \tilde{\varphi}_{h}, \psi_{h}, \bar{\psi}_{h}, \tilde{\psi}_{h}\right)=0 .
$$

The exact solution $p, q, r$ also satisfies

$$
\begin{aligned}
& \mathcal{A}_{i, j}\left(p, q, r, p, q, r ; \varphi_{h}, \bar{\varphi}_{h}, \tilde{\varphi}_{h}, \psi_{h}, \bar{\psi}_{h}, \tilde{\psi}_{h}\right)=0, \\
& \quad \forall i, j \text { and } \forall\left(\varphi_{h}, \bar{\varphi}_{h}, \tilde{\varphi}_{h}\right) \in\left[V_{h}\right]^{3},\left(\psi_{h}, \bar{\psi}_{h}, \tilde{\psi}_{h}\right) \in\left[W_{h}\right]^{3} .
\end{aligned}
$$

Subtracting (3.42) from (3.43), we obtain the error equation

$$
\begin{aligned}
& \mathcal{A}_{i, j}\left(p-p_{h}, q-q_{h}, r-r_{h}, p-u_{h}, q-v_{h}, r-w_{h} ; \varphi_{h}, \bar{\varphi}_{h}, \tilde{\varphi}_{h}, \psi_{h}, \bar{\psi}_{h}, \tilde{\psi}_{h}\right)=0, \\
& \forall i, j \text { and } \forall\left(\varphi_{h}, \bar{\varphi}_{h}, \tilde{\varphi}_{h}\right) \in\left[V_{h}\right]^{3},\left(\psi_{h}, \bar{\psi}_{h}, \tilde{\psi}_{h}\right) \in\left[W_{h}\right]^{3} .
\end{aligned}
$$

We now define the special local coupled projection for the two dimensional problem. For any functions $p, q, r \in H^{1}(\Omega)$, define the following coupled projection $\mathbb{P}^{\star}:\left[H^{1}(\Omega)\right]^{3} \rightarrow\left[V_{h}\right]^{3}, \mathbb{P}^{\star}(p, q, r):=\left(\mathbb{P}^{1, \star} p, \mathbb{P}^{2, \star} q, \mathbb{P}^{3, \star} r\right)$ such that

$$
\begin{array}{rlrl}
\int_{K_{i, j}} \mathbb{P}^{1, \star} p \mathrm{~d} x \mathrm{~d} y & =\int_{K_{i, j}} p \mathrm{~d} x \mathrm{~d} y, & \\
\int_{K_{i, j}} \mathbb{P}^{2, \star} q \mathrm{~d} x \mathrm{~d} y & =\int_{K_{i, j}} q \mathrm{~d} x \mathrm{~d} y, & \\
\int_{K_{i, j}} \mathbb{P}^{3, \star} r \mathrm{~d} x \mathrm{~d} y & =\int_{K_{i, j}} r \mathrm{~d} x \mathrm{~d} y, & \\
\widetilde{P}_{h}^{1}\left(\mathbb{P}^{1, \star} p, \mathbb{P}^{2, \star} q, \mathbb{P}^{3, \star} r ; \varphi_{h}\right)_{i, j} & =\widetilde{P}_{h}^{1}\left(p, q, r ; \varphi_{h}\right)_{i, j}, & & \forall \varphi_{h} \in P^{k}\left(K_{i, j}\right), \\
{\widetilde{P_{h}}}^{2}\left(\mathbb{P}^{2, \star} q, \mathbb{P}^{1, \star} p ; \bar{\varphi}_{h}\right)_{i, j} & =\widetilde{P}_{h}^{2}\left(q, p ; \bar{\varphi}_{h}\right)_{i, j}, & & \forall \varphi_{h} \in P^{k}\left(K_{i, j}\right), \\
{\widetilde{P_{h}}}^{2}\left(\mathbb{P}^{3, \star} r, \mathbb{P}^{1, \star} p ; \bar{\varphi}_{h}\right)_{i, j} & =\widetilde{P}_{h}{ }^{2}\left(r, p ; \bar{\varphi}_{h}\right)_{i, j}, & & \forall \varphi_{h} \in P^{k}\left(K_{i, j}\right),
\end{array}
$$


where ${\widetilde{P_{h}}}^{1}$ and ${\widetilde{P_{h}}}^{2}$ are given as

$$
\begin{aligned}
& \widetilde{P}_{h}^{1}\left(p, q, r ; \varphi_{h}\right)_{i, j}=\frac{1}{\tau_{\max }}\left(\int_{K_{i, j}^{1}} p\left(x+h_{x} / 2, y+h_{y} / 2\right) \varphi_{h} \mathrm{~d} x \mathrm{~d} y+\int_{K_{i, j}^{2}} p\left(x-h_{x} / 2, y+h_{y} / 2\right) \varphi_{h} \mathrm{~d} x \mathrm{~d} y\right. \\
& +\int_{K_{i, j}^{3}} p\left(x+h_{x} / 2, y-h_{y} / 2\right) \varphi_{h} \mathrm{~d} x \mathrm{~d} y+\int_{K_{i, j}^{4}} p\left(x-h_{x} / 2, y-h_{y} / 2\right) \varphi_{h} \mathrm{~d} x \mathrm{~d} y \\
& \left.-\int_{K_{i, j}} p(x, y) \varphi_{h} \mathrm{~d} x \mathrm{~d} y\right) \\
& +\int_{K_{i, j}^{1}} q\left(x+h_{x} / 2, y+h_{y} / 2\right)\left(\varphi_{h}\right)_{x} \mathrm{~d} x \mathrm{~d} y+\int_{K_{i, j}^{2}} q\left(x-h_{x} / 2, y+h_{y} / 2\right)\left(\varphi_{h}\right)_{x} \mathrm{~d} x \mathrm{~d} y \\
& +\int_{K_{i, j}^{3}} q\left(x+h_{x} / 2, y-h_{y} / 2\right)\left(\varphi_{h}\right)_{x} \mathrm{~d} x \mathrm{~d} y+\int_{K_{i, j}^{4}} q\left(x-h_{x} / 2, y-h_{y} / 2\right)\left(\varphi_{h}\right)_{x} \mathrm{~d} x \mathrm{~d} y \\
& +\int_{K_{i, j}^{1}} r\left(x+h_{x} / 2, y+h_{y} / 2\right)\left(\varphi_{h}\right)_{y} \mathrm{~d} x \mathrm{~d} y+\int_{K_{i, j}^{2}} r\left(x-h_{x} / 2, y+h_{y} / 2\right)\left(\varphi_{h}\right)_{y} \mathrm{~d} x \mathrm{~d} y \\
& +\int_{K_{i, j}^{3}} r\left(x+h_{x} / 2, y-h_{y} / 2\right)\left(\varphi_{h}\right)_{y} \mathrm{~d} x \mathrm{~d} y+\int_{K_{i, j}^{4}} r\left(x-h_{x} / 2, y-h_{y} / 2\right)\left(\varphi_{h}\right)_{y} \mathrm{~d} x \mathrm{~d} y \\
& -\int_{y_{j-\frac{1}{2}}}^{y_{j}} q\left(x_{i}, y+h_{y} / 2\right)\left(\varphi_{h}\left(x_{i+\frac{1}{2}}^{-}, y\right)-\varphi_{h}\left(x_{i-\frac{1}{2}}^{+}, y\right)\right) \mathrm{d} y \\
& -\int_{y_{j}}^{y_{j+\frac{1}{2}}} q\left(x_{i}, y-h_{y} / 2\right)\left(\varphi_{h}\left(x_{i+\frac{1}{2}}^{-}, y\right)-\varphi_{h}\left(x_{i-\frac{1}{2}}^{+}, y\right)\right) \mathrm{d} y \\
& -\int_{x_{i-\frac{1}{2}}}^{x_{i}} r\left(x+h_{x} / 2, y_{j}\right)\left(\varphi_{h}\left(x, y_{j+\frac{1}{2}}^{-}\right)-\varphi_{h}\left(x, y_{j-\frac{1}{2}}^{+}\right)\right) \mathrm{d} x \\
& -\int_{x_{i}}^{x_{i+\frac{1}{2}}} r\left(x-h_{x} / 2, y_{j}\right)\left(\varphi_{h}\left(x, y_{j+\frac{1}{2}}^{-}\right)-\varphi_{h}\left(x, y_{j-\frac{1}{2}}^{+}\right)\right) \mathrm{d} x, \\
& {\widetilde{P_{h}}}^{2}\left(q, p ; \bar{\varphi}_{h}\right)_{i, j}=\frac{1}{\tau_{\max }}\left(\int_{K_{i, j}^{1}} q\left(x+h_{x} / 2, y+h_{y} / 2\right) \bar{\varphi}_{h} \mathrm{~d} x \mathrm{~d} y+\int_{K_{i, j}^{2}} q\left(x-h_{x} / 2, y+h_{y} / 2\right) \bar{\varphi}_{h} \mathrm{~d} x \mathrm{~d} y\right. \\
& +\int_{K_{i, j}^{3}} q\left(x+h_{x} / 2, y-h_{y} / 2\right) \bar{\varphi}_{h} \mathrm{~d} x \mathrm{~d} y+\int_{K_{i, j}^{4}} q\left(x-h_{x} / 2, y-h_{y} / 2\right) \bar{\varphi}_{h} \mathrm{~d} x \mathrm{~d} y \\
& \left.-\int_{K_{i, j}} q(x, y) \bar{\varphi}_{h} \mathrm{~d} x \mathrm{~d} y\right) \\
& +\int_{K_{i, j}^{1}} p\left(x+h_{x} / 2, y+h_{y} / 2\right)\left(\bar{\varphi}_{h}\right)_{x} \mathrm{~d} x \mathrm{~d} y+\int_{K_{i, j}^{2}} p\left(x-h_{x} / 2, y+h_{y} / 2\right)\left(\bar{\varphi}_{h}\right)_{x} \mathrm{~d} x \mathrm{~d} y \\
& +\int_{K_{i, j}^{3}} p\left(x+h_{x} / 2, y-h_{y} / 2\right)\left(\bar{\varphi}_{h}\right)_{x} \mathrm{~d} x \mathrm{~d} y+\int_{K_{i, j}^{4}} p\left(x-h_{x} / 2, y-h_{y} / 2\right)\left(\bar{\varphi}_{h}\right)_{x} \mathrm{~d} x \mathrm{~d} y \\
& -\int_{y_{j}}^{y_{j+\frac{1}{2}}} p\left(x_{i}, y-h_{y} / 2\right)\left(\bar{\varphi}_{h}\left(x_{i+\frac{1}{2}}^{-}, y\right)-\bar{\varphi}_{h}\left(x_{i-\frac{1}{2}}^{+}, y\right)\right) \mathrm{d} y \\
& -\int_{y_{j}}^{y_{j+\frac{1}{2}}} p\left(x_{i}, y-h_{y} / 2\right)\left(\bar{\varphi}_{h}\left(x_{i+\frac{1}{2}}^{-}, y\right)-\bar{\varphi}_{h}\left(x_{i-\frac{1}{2}}^{+}, y\right)\right) \mathrm{d} y,
\end{aligned}
$$

where $K_{i, j}^{1}=\left(x_{i-\frac{1}{2}}, x_{i}\right) \times\left(y_{j-\frac{1}{2}}, y_{j}\right), K_{i, j}^{2}=\left(x_{i}, x_{i+\frac{1}{2}}\right) \times\left(y_{j-\frac{1}{2}}, y_{j}\right), K_{i, j}^{3}=\left(x_{i-\frac{1}{2}}, x_{i}\right) \times\left(y_{j}, y_{j+\frac{1}{2}}\right)$, $K_{i, j}^{4}=\left(x_{i}, x_{i+\frac{1}{2}}\right) \times\left(y_{j}, y_{j+\frac{1}{2}}\right)$. 
Similarly, we can define $\mathbb{Q}_{h}^{\star}(p, q, r):=\left(\mathbb{Q}_{h}^{1, \star} p, \mathbb{Q}_{h}^{2, \star} q, \mathbb{Q}_{h}^{3, \star} r\right) \in\left[W_{h}\right]^{3}$. Next, we prove the projections $\mathbb{P}_{h}^{\star}$ and $Q_{h}^{\star}$ are well defined. Similar to the argument in the one dimensional case, we need to show the existence and uniqueness of projections on the reference cell $[-1,1]^{2}$. In this case, $\tau_{\max }=2 \alpha, \alpha$ is a constant. The following lemma implies the projection $\mathbb{P}_{h}^{\star}$ is well defined.

Lemma 3.8. The projection $\mathbb{P}_{h}^{\star}$ defined by (3.45a)-(3.45f) on the cell $[-1,1]^{2}$ exists and is unique for any functions $p, q, r \in H^{1}(\Omega)$, and the projection is bounded in the $L^{\infty}$ norm, i.e.

$$
\left\|\mathbb{P}_{h}^{1, \star} p\right\|_{\infty}+\left\|\mathbb{P}_{h}^{2, \star} q\right\|_{\infty}+\left\|\mathbb{P}_{h}^{3, \star} r\right\|_{\infty} \leq C(k)\left(\|p\|_{\infty}+\|q\|_{\infty}+\|r\|_{\infty}\right) .
$$

where $C(k)$ is a constant that only depends on $k$ but is independent of $p, q, r$.

Proof. We give the proof of this lemma in the Appendix A.4.

The standard approximation theory, Theorem 3.1.5 in [5] implies, for smooth functions $p, q, r$,

$$
\begin{aligned}
&\left\|\mathbb{P}_{h}^{1, \star} p-p\right\|+\left\|\mathbb{P}_{h}^{2, \star} q-q\right\|+\left\|\mathbb{P}_{h}^{3, \star} r-r\right\| \lesssim h^{k+1}, \\
&\left\|\mathbb{P}_{h}^{1, \star} p-p\right\|_{\Gamma_{h}}+\left\|\mathbb{P}_{h}^{2, \star} q-q\right\|_{\Gamma_{h}}+\left\|\mathbb{P}_{h}^{3, \star} r-r\right\|_{\Gamma_{h}} \lesssim h^{k+\frac{1}{2}} .
\end{aligned}
$$

Analogous to Lemma 3.3 in the one dimensional case, we have the following lemma.

Lemma 3.9. Assume that $(p, q, r)=\left(a x^{k+1-\ell} y^{\ell}, b x^{k+1-\ell} y^{\ell}, c x^{k+1-\ell} y^{\ell}\right)$, where $a, b, c$ are constants, $\ell=0, \ldots, k+1$. Then we have the projections $\mathbb{P}_{h}^{\star}(p, q, r)=\left(\mathbb{P}_{h}^{1, \star} p, \mathbb{P}_{h}^{2, \star} q, \mathbb{P}_{h}^{3, \star} r\right), \mathbb{Q}_{h}^{\star}(p, q, r)=$ $\left(\mathbb{Q}_{h}^{1, \star} p, \mathbb{Q}_{h}^{2, \star} q, \mathbb{Q}_{h}^{3, \star} r\right)$, and we have

$$
\begin{aligned}
\left(p(x, y)-\mathbb{P}_{h}^{1, \star} p(x, y), q(x, y)-\mathbb{P}_{h}^{2, \star} q(x, y), r(x, y)-\mathbb{P}_{h}^{3, \star} r(x, y)\right) & \left(p\left(x \mp h_{x} / 2, y \mp h_{y} / 2\right)-\mathbb{Q}_{h}^{1, \star} p\left(x \mp h_{x} / 2, y \mp h_{y} / 2\right),\right. \\
& q\left(x \mp h_{x} / 2, y \mp h_{y} / 2\right)-\mathbb{Q}_{h}^{2, \star} q\left(x \mp h_{x} / 2, y \mp h_{y} / 2\right), \\
& \left.r\left(x \mp h_{x} / 2, y \mp h_{y} / 2\right)-\mathbb{Q}_{h}^{3, \star} r\left(x \mp h_{x} / 2, y \mp h_{y} / 2\right)\right) \\
= & \left(p\left(x \mp h_{x} / 2, y \pm h_{y} / 2\right)-\mathbb{Q}_{h}^{1, \star} p\left(x \mp h_{x} / 2, y \pm h_{y} / 2\right),\right. \\
& q\left(x \mp h_{x} / 2, y \pm h_{y} / 2\right)-\mathbb{Q}_{h}^{2, \star} q\left(x \mp h_{x} / 2, y \pm h_{y} / 2\right), \\
& \left.r\left(x \mp h_{x} / 2, y \pm h_{y} / 2\right)-\mathbb{Q}_{h}^{3, \star} r\left(x \mp h_{x} / 2, y \pm h_{y} / 2\right)\right) .
\end{aligned}
$$

Proof. We provide the proof of this lemma in the Appendix A.5.

Again, using the above lemma we can show that $\mathbb{P}_{h}^{\star}$ and $\mathbb{Q}_{h}^{\star}$ satisfy the following superconvergence property.

Lemma 3.10. Assume that $(p, q, r)=\left(a x^{k+1-\ell} y^{\ell}, b x^{k+1-\ell} y^{\ell}, c x^{k+1-\ell} y^{\ell}\right), \ell=0,1, \ldots, k+1$. Let $\left(p_{I}, q_{I}, r_{I}\right)=$ $\left(\mathbb{P}^{1, \star} p, \mathbb{P}^{2, \star} q, \mathbb{P}^{3, \star} r\right),\left(u_{I}, v_{I}, w_{I}\right)=\left(\mathbb{Q}^{1, \star} p, \mathbb{Q}^{2, \star} q, \mathbb{Q}^{3, \star} r\right)$, then

$$
\begin{array}{ll}
\mathcal{B}_{1}\left(p-u_{I}, p-p_{I}, q-v_{I}, r-w_{I} ; \varphi_{h}\right)_{i, j}=0, & \forall \varphi_{h} \in P^{k}\left(K_{i, j}\right), \\
\mathcal{B}_{1}\left(p-p_{I}, p-u_{I}, q-q_{I}, r-r_{I} ; \psi_{h}\right)_{i, j}=0, & \forall \psi_{h} \in P^{k}\left(K_{i+\frac{1}{2}, j+\frac{1}{2}}\right), \\
\mathcal{B}_{2}\left(q-v_{I}, q-q_{I}, p-u_{I} ; \bar{\varphi}_{h}\right)_{i, j}=0, & \forall \bar{\varphi}_{h} \in P^{k}\left(K_{i, j}\right), \\
\mathcal{B}_{2}\left(q-q_{I}, q-v_{I}, p-p_{I} ; \bar{\psi}_{h}\right)_{i+\frac{1}{2}, j+\frac{1}{2}}=0, & \forall \bar{\psi}_{h} \in P^{k}\left(K_{i+\frac{1}{2}, j+\frac{1}{2}}\right), \\
\mathcal{B}_{3}\left(r-w_{I}, r-r_{I}, p-u_{I} ; \tilde{\varphi}_{h}\right)_{i, j}=0, & \forall \tilde{\varphi}_{h} \in P^{k}\left(K_{i, j}\right), \\
\mathcal{B}_{3}\left(r-r_{I}, r-w_{I}, p-p_{I} ; \tilde{\psi}_{h}\right)_{i+\frac{1}{2}, j+\frac{1}{2}}=0, & \forall \tilde{\psi}_{h} \in P^{k}\left(K_{i+\frac{1}{2}, j+\frac{1}{2}}\right) .
\end{array}
$$


Proof. We provide the proof of this lemma in the Appendix A.6.

Next, we can use above lemmas to prove our main result in the following theorem.

Theorem 3.11 (Error estimates). Suppose that $p, q, r \in H^{k+2}(\Omega)$ are exact solutions of the equation (3.37) with smooth initial condition $p(\cdot, 0), q(\cdot, 0), r(\cdot, 0) \in H^{k+2}(\Omega)$. The numerical solutions $p_{h}, q_{h}, r_{h}, u_{h}, v_{h}, w_{h}$ of the central DG scheme (3.38) satisfy the following $L^{2}$ error estimates

$$
\left\|p-p_{h}\right\|^{2}+\left\|q-q_{h}\right\|^{2}+\left\|r-r_{h}\right\|^{2}+\left\|p-u_{h}\right\|^{2}+\left\|q-v_{h}\right\|^{2}+\left\|r-w_{h}\right\|^{2} \lesssim h^{2 k+2} .
$$

Proof. In the error equation (3.44), we take

$$
\begin{aligned}
& \varphi_{h}=p_{h}-\mathbb{P}_{h}^{1, \star} p, \quad \bar{\varphi}_{h}=q_{h}-\mathbb{P}_{h}^{2, \star} q, \quad \tilde{\varphi}_{h}=r_{h}-\mathbb{P}_{h}^{3, \star} r, \\
& \psi_{h}=u_{h}-\mathbb{Q}_{h}^{1, \star} p, \quad \bar{\psi}_{h}=v_{h}-\mathbb{Q}_{h}^{2, \star} q, \quad \tilde{\psi}_{h}=w_{h}-\mathbb{Q}_{h}^{3, \star} r,
\end{aligned}
$$

and define

$$
\begin{aligned}
& p_{e}=p-\mathbb{P}_{h}^{1, \star} p, \quad q_{e}=q-\mathbb{P}_{h}^{2, \star} q, \quad r_{e}=r-\mathbb{P}_{h}^{3, \star} r, \\
& u_{e}=p-\mathbb{Q}_{h}^{1, \star} p, \quad v_{e}=q-\mathbb{Q}_{h}^{2, \star} q, \quad w_{e}=r-\mathbb{Q}_{h}^{3, \star} r .
\end{aligned}
$$

Then we obtain

$$
\begin{aligned}
\mathcal{A}_{i, j} & \left(\varphi_{h}, \bar{\varphi}_{h}, \tilde{\varphi}_{h}, \psi_{h}, \bar{\psi}_{h}, \tilde{\psi}_{h} ; \varphi_{h}, \bar{\varphi}_{h}, \tilde{\varphi}_{h}, \psi_{h}, \bar{\psi}_{h}, \tilde{\psi}_{h}\right) \\
& =\mathcal{A}_{i, j}\left(p_{e}, q_{e}, r_{e}, u_{e}, v_{e}, w_{e} ; \varphi_{h}, \bar{\varphi}_{h}, \tilde{\varphi}_{h}, \psi_{h}, \bar{\psi}_{h}, \tilde{\psi}_{h}\right) .
\end{aligned}
$$

For the left-hand side of (3.58), we mimic the proof of the $L^{2}$ stability to conclude

$$
\begin{aligned}
\sum_{i, j} \mathcal{A}_{i, j} & \left(\varphi_{h}, \bar{\varphi}_{h}, \tilde{\varphi}_{h}, \psi_{h}, \bar{\psi}_{h}, \tilde{\psi}_{h} ; \varphi_{h}, \bar{\varphi}_{h}, \tilde{\varphi}_{h}, \psi_{h}, \bar{\psi}_{h}, \tilde{\psi}_{h}\right) \\
= & \frac{1}{2} \frac{\mathrm{d}}{\mathrm{d} t} \int_{\Omega} \varphi_{h}^{2}+\bar{\varphi}_{h}^{2}+\tilde{\varphi}_{h}^{2}+\psi_{h}^{2}+\bar{\psi}_{h}^{2}+\tilde{\psi}_{h}^{2} \mathrm{~d} x \mathrm{~d} y \\
& +\frac{1}{\tau_{\max }} \int_{\Omega}\left(\varphi_{h}-\psi_{h}\right)^{2}+\left(\bar{\varphi}_{h}-\bar{\psi}_{h}\right)^{2}+\left(\tilde{\varphi}_{h}-\tilde{\psi}_{h}\right)^{2} \mathrm{~d} x \mathrm{~d} y
\end{aligned}
$$

For the right-hand side of (3.58), we rewrite it as a sum of seven terms

$$
\mathcal{A}_{i, j}\left(p_{e}, q_{e}, r_{e}, u_{e}, v_{e}, w_{e} ; \varphi_{h}, \bar{\varphi}_{h}, \tilde{\varphi}_{h}, \psi_{h}, \bar{\psi}_{h}, \tilde{\psi}_{h}\right)=\sum_{\ell=1}^{7} \mathcal{A}_{i, j}^{\ell},
$$

where $A_{i, j}^{1}, \ldots, A_{i, j}^{7}$ are given as

$$
\begin{aligned}
\mathcal{A}_{i, j}^{1}= & \int_{K_{i, j}} \partial_{t} p_{e} \varphi_{h} \mathrm{~d} x \mathrm{~d} y+\int_{K_{i, j}} \partial_{t} q_{e} \bar{\varphi}_{h} \mathrm{~d} x \mathrm{~d} y+\int_{K_{i, j}} \partial_{t} r_{e} \tilde{\varphi}_{h} \mathrm{~d} x \mathrm{~d} y \\
& +\int_{K_{i+\frac{1}{2}, j+\frac{1}{2}}} \partial_{t} u_{e} \psi_{h} \mathrm{~d} x \mathrm{~d} y+\int_{K_{i+\frac{1}{2}, j+\frac{1}{2}}} \partial_{t} v_{e} \bar{\psi}_{h} \mathrm{~d} x \mathrm{~d} y+\int_{K_{i+\frac{1}{2}, j+\frac{1}{2}}} \partial_{t} w_{e} \tilde{\psi}_{h} \mathrm{~d} x \mathrm{~d} y, \\
\mathcal{A}_{i, j}^{2}= & -\mathcal{B}_{1}\left(u_{e}, p_{e}, v_{e}, w_{e} ; \varphi_{h}\right)_{i, j}, \mathcal{A}_{i, j}^{3}=-\mathcal{B}_{2}\left(v_{e}, q_{e}, u_{e} ; \bar{\varphi}_{h}\right)_{i, j}, \mathcal{A}_{i, j}^{4}=-\mathcal{B}_{3}\left(w_{e}, r_{e}, u_{e} ; \tilde{\varphi}_{h}\right)_{i, j}, \\
\mathcal{A}_{i, j}^{5}= & -\mathcal{B}_{1}\left(p_{e}, u_{e}, q_{e}, r_{e} ; \psi_{h}\right)_{i+\frac{1}{2}, j+\frac{1}{2}}, \quad \mathcal{A}_{i, j}^{6}=-\mathcal{B}_{2}\left(q_{e}, v_{e}, p_{e} ; \bar{\psi}_{h}\right)_{i+\frac{1}{2}, j+\frac{1}{2}}, \\
\mathcal{A}_{i, j}^{7}= & -\mathcal{B}_{3}\left(r_{e}, w_{e}, p_{e} ; \tilde{\psi}_{h}\right)_{i+\frac{1}{2}, j+\frac{1}{2}} .
\end{aligned}
$$


Now we proceed to estimate each term of $\mathcal{A}_{i, j}^{1}, \ldots, \mathcal{A}_{i, j}^{7}$ separately. For $\mathcal{A}_{i, j}^{1}$, we use the optimal error estimates of the projections to obtain

$$
\sum_{i, j}\left|\mathcal{A}_{i, j}^{1}\right| \lesssim h^{k+1}\left(\left\|\varphi_{h}\right\|^{2}+\left\|\bar{\varphi}_{h}\right\|^{2}+\left\|\tilde{\varphi}_{h}\right\|^{2}+\left\|\psi_{h}\right\|^{2}+\left\|\bar{\psi}_{h}\right\|^{2}+\left\|\tilde{\psi}_{h}\right\|^{2}\right)^{\frac{1}{2}} .
$$

Next we give the details of the estimate for $\mathcal{A}_{i, j}^{2}$ as an example. For $\mathcal{A}_{i, j}^{\ell}, \ell=3, \ldots, 7$, the analysis is similar. We use Lemma 3.10 and the Bramble-Hilbert lemma in [5] to obtain

$$
\begin{aligned}
\mathcal{B}_{1}\left(u_{e}, p_{e}, v_{e}, w_{e} ; \varphi_{h}\right)_{i, j}= & \mathcal{B}_{1}\left(p-\chi_{p}, p-\chi_{p}, q-\chi_{q}, r-\chi_{r} ; \varphi_{h}\right)_{i, j} \\
& +\mathcal{B}_{1}\left(\chi_{p}-\mathbb{Q}_{h}^{1, \star} \chi_{p}, \chi_{p}-\mathbb{P}_{h}^{1, \star} \chi_{p}, \chi_{q}-\mathbb{Q}_{h}^{2, \star} \chi_{q}, \chi_{r}-\mathbb{Q}_{h}^{3, \star} \chi_{r} ; \varphi_{h}\right)_{i, j} \\
& +\mathcal{B}_{1}\left(\mathbb{Q}_{h}^{1, \star}\left(\chi_{p}-p\right), \mathbb{P}_{h}^{1, \star}\left(\chi_{p}-p\right), \mathbb{Q}_{h}^{2, \star}\left(q-\chi_{q}\right), \mathbb{Q}_{h}^{3, \star}\left(\chi_{r}-r\right) ; \varphi_{h}\right)_{i, j} \\
= & \mathcal{B}_{1}\left(p-\chi_{p}, p-\chi_{p}, q-\chi_{q}, r-\chi_{r} ; \varphi_{h}\right)_{i, j} \\
& +\mathcal{B}_{1}\left(\mathbb{Q}_{h}^{1, \star}\left(\chi_{p}-p\right), \mathbb{P}_{h}^{1, \star}\left(\chi_{p}-p\right), \mathbb{Q}_{h}^{2, \star}\left(q-\chi_{q}\right), \mathbb{Q}_{h}^{3, \star}\left(\chi_{r}-r\right) ; \varphi_{h}\right)_{i, j} \\
& \lesssim h^{k+1}\left(\|p\|_{k+2, D_{i, j}}+\|q\|_{k+2, D_{i, j}}+\|r\|_{k+2, D_{i, j}}\right)\left\|\varphi_{h}\right\|_{0, K_{i, j}},
\end{aligned}
$$

where $D_{i, j}=K_{i-\frac{1}{2}, j-\frac{1}{2}} \bigcup K_{i-\frac{1}{2}, j+\frac{1}{2}} \bigcup K_{i+\frac{1}{2}, j-\frac{1}{2}} \bigcup K_{i+\frac{1}{2}, j+\frac{1}{2}}$ and $\left(\chi_{p}, \chi_{q}, \chi_{r}\right)$ are arbitrary functions in $\left[P^{k+1}\left(D_{i, j}\right)\right]^{3}$. Thus summing (3.62) over $i, j$, we have

$$
\begin{aligned}
\sum_{i, j}\left|\mathcal{A}_{i, j}^{2}\right| & =\sum_{i, j}\left|\mathcal{B}_{1}\left(u_{e}, p_{e}, v_{e}, w_{e} ; \varphi_{h}\right)_{i, j}\right| \\
& \lesssim h^{k+1}\left(\|p\|_{k+2}^{2}+\|q\|_{k+2}^{2}+\|r\|_{k+2}^{2}\right)^{\frac{1}{2}}\left\|\varphi_{h}\right\| .
\end{aligned}
$$

Similarly, we also have the estimates for $\mathcal{A}_{i, j}^{3}, \ldots, \mathcal{A}_{i, j}^{7}$.

$$
\begin{aligned}
& \sum_{i, j}\left|\mathcal{A}_{i, j}^{3}\right| \lesssim h^{k+1}\left(\|p\|_{k+2}^{2}+\|q\|_{k+2}^{2}+\|r\|_{k+2}^{2}\right)^{\frac{1}{2}}\left\|\bar{\varphi}_{h}\right\|, \\
& \sum_{i, j}\left|\mathcal{A}_{i, j}^{4}\right| \lesssim h^{k+1}\left(\|p\|_{k+2}^{2}+\|q\|_{k+2}^{2}+\|r\|_{k+2}^{2}\right)^{\frac{1}{2}}\left\|\tilde{\varphi}_{h}\right\|, \\
& \sum_{i, j}\left|\mathcal{A}_{i, j}^{5}\right| \lesssim h^{k+1}\left(\|p\|_{k+2}^{2}+\|q\|_{k+2}^{2}+\|r\|_{k+2}^{2}\right)^{\frac{1}{2}}\left\|\psi_{h}\right\|, \\
& \sum_{i, j}\left|\mathcal{A}_{i, j}^{6}\right| \lesssim h^{k+1}\left(\|p\|_{k+2}^{2}+\|q\|_{k+2}^{2}+\|r\|_{k+2}^{2}\right)^{\frac{1}{2}}\left\|\bar{\psi}_{h}\right\|, \\
& \sum_{i, j}\left|\mathcal{A}_{i, j}^{7}\right| \lesssim h^{k+1}\left(\|p\|_{k+2}^{2}+\|q\|_{k+2}^{2}+\|r\|_{k+2}^{2}\right)^{\frac{1}{2}}\left\|\tilde{\psi}_{h}\right\| .
\end{aligned}
$$

Substituting the estimates (3.61), (3.63) and (3.64) into the left-hand side of (3.58), and combining with (3.59) we have

$$
\frac{1}{2} \frac{\mathrm{d}}{\mathrm{d} t} \int_{\Omega} \varphi_{h}^{2}+\bar{\varphi}_{h}^{2}+\tilde{\varphi}_{h}^{2}+\psi_{h}^{2}+\bar{\psi}_{h}^{2}+\tilde{\psi}_{h}^{2} \mathrm{~d} x \mathrm{~d} y \lesssim h^{k+1}\left(\int_{\Omega} \varphi_{h}^{2}+\bar{\varphi}_{h}^{2}+\tilde{\varphi}_{h}^{2}+\psi_{h}^{2}+\bar{\psi}_{h}^{2}+\tilde{\psi}_{h}^{2} \mathrm{~d} x \mathrm{~d} y\right)^{\frac{1}{2}} .
$$

Finally, integrate the above inequality over $[0, t]$, together with the initial projection error implies the designed result (3.55). 


\section{Numerical EXAmples}

In this section, we present some numerical examples to validate our theoretical results. First, we will test the accuracy of our algorithm for one and two dimensional problems, and then we will take a long time simulation to see how the $L^{2}$ error and energy evolve against time. Two kinds of time-stepping methods are adopted in this section. For the test of accuracy, we take the classical fourth order Runge-Kutta method as the time-stepping method, see e.g. [2]. For the long time simulation, we use the ninth order strong stability preserving RungeKutta (SSPRK) method [16], to reduce the error from the temporal discretization [3]. The CFL condition is $\tau=O(h)$, where $\tau$ is the temporal step size and $h$ is the maximum spatial step size.

\subsection{Accuracy test}

Example 4.1. Consider the two way wave equation (3.1). We take the initial conditions

$$
p_{0}(x)=\sin (x), \quad q_{0}(x)=-\cos (x),
$$

such that the exact solutions are given as

$$
p(x, t)=(\sin (c t)+\cos (c t)) \sin (x), \quad q(x, t)=(\sin (c t)-\cos (c t)) \cos (x),
$$

where the sound speed is $c=1.2$. The computation domain is $(0,2 \pi)$, and the final time is $T=1.3$. Table 1 shows the designed orders of accuracy.

Example 4.2. Consider the two dimensional wave equation (3.37). We take the following initial conditions

$$
p_{0}(x, y)=\sin (x) \sin (y), \quad q_{0}(x, y)=-\frac{1}{\sqrt{2}} \cos (x) \sin (y), \quad r_{0}(x, y)=-\frac{1}{\sqrt{2}} \sin (x) \cos (y) .
$$

Then the exact solutions are

$$
\begin{aligned}
& p(x, y, t)=(\sin (\sqrt{2} c t)+\cos (\sqrt{2} c t)) \sin (x) \sin (y), \\
& q(x, y, t)=\frac{1}{\sqrt{2}}(\sin (\sqrt{2} c t)-\cos (\sqrt{2} c t)) \cos (x) \sin (y), \\
& r(x, y, t)=\frac{1}{\sqrt{2}}(\sin (\sqrt{2} c t)-\cos (\sqrt{2} c t)) \sin (x) \cos (y),
\end{aligned}
$$

where the sound speed is $c=1.2$. The computational domain is $(0,2 \pi) \times(0,2 \pi)$, and the final time is $T=0.6$. Similarly, Tables 2 and 3 show the designed orders of accuracy.

\subsection{Long time behaviors}

In this subsection, we study the long time behavior of the DG scheme (3.2) in Example 4.1. We study the time history of the $L^{2}$ error and energy of the numerical solution up to $T=3000$. We consider $k=0,1,2$ in the finite element spaces $V_{h}$ and $W_{h}$ on the fixed uniform mesh $h=\pi / 32$. The $L^{2}$ error is

$$
\left(\left\|p-p_{h}\right\|^{2}+\left\|q-q_{h}\right\|^{2}+\left\|p-r_{h}\right\|^{2}+\left\|q-s_{h}\right\|^{2}\right)^{\frac{1}{2}},
$$

and the energy is

$$
\left\|p_{h}\right\|^{2}+\left\|q_{h}\right\|^{2}+\left\|r_{h}\right\|^{2}+\left\|s_{h}\right\|^{2} .
$$

In the simulation, we make the comparisons between different $\alpha$ in $\tau_{\max }$, to see how $\alpha$ will affect the long time behavior of the numerical solutions. From Figure 2, we can see that for the $P^{0}$ element, the numerical 
TABLE 1. Errors and convergence orders produced by the scheme (3.2) with $k=0,1,2,3$ in Example 4.1. The final time is $T=1.3$.

\begin{tabular}{clllllllll}
\hline \hline \multirow{2}{*}{$N$} & \multicolumn{2}{c}{$\left\|p-p_{h}\right\|$} & \multicolumn{2}{c}{$\left\|q-q_{h}\right\|$} & \multicolumn{2}{c}{$\left\|p-r_{h}\right\|$} & \multicolumn{2}{c}{$\left\|q-s_{h}\right\|$} \\
\cline { 2 - 10 } & & $L^{2}$ error & order & $L^{2}$ error & order & $L^{2}$ error & order & $L^{2}$ error & order \\
\hline \multirow{6}{*}{$P^{0}$} & 16 & $8.90 \mathrm{E}-02$ & - & $9.33 \mathrm{E}-02$ & - & $8.90 \mathrm{E}-02$ & - & $9.33 \mathrm{E}-02$ & - \\
& 32 & $4.55 \mathrm{E}-02$ & 0.97 & $4.61 \mathrm{E}-02$ & 1.02 & $4.55 \mathrm{E}-02$ & 0.97 & $4.61 \mathrm{E}-02$ & 1.02 \\
& 64 & $2.30 \mathrm{E}-02$ & 0.98 & $2.29 \mathrm{E}-02$ & 1.01 & $2.30 \mathrm{E}-02$ & 0.98 & $2.29 \mathrm{E}-02$ & 1.01 \\
& 128 & $1.16 \mathrm{E}-02$ & 0.99 & $1.14 \mathrm{E}-02$ & 1.01 & $1.16 \mathrm{E}-02$ & 0.99 & $1.14 \mathrm{E}-02$ & 1.01 \\
& 256 & $5.79 \mathrm{E}-03$ & 1.00 & $5.70 \mathrm{E}-03$ & 1.00 & $5.79 \mathrm{E}-03$ & 1.00 & $5.70 \mathrm{E}-03$ & 1.00 \\
& 512 & $2.90 \mathrm{E}-03$ & 1.00 & $2.85 \mathrm{E}-03$ & 1.00 & $2.90 \mathrm{E}-03$ & 1.00 & $2.85 \mathrm{E}-03$ & 1.00 \\
\hline \multirow{6}{*}{$P^{1}$} & 16 & $4.55 \mathrm{E}-03$ & - & $1.20 \mathrm{E}-02$ & - & $4.55 \mathrm{E}-03$ & - & $1.20 \mathrm{E}-02$ & - \\
& 32 & $1.05 \mathrm{E}-03$ & 2.11 & $3.11 \mathrm{E}-03$ & 1.94 & $1.05 \mathrm{E}-03$ & 2.11 & $3.11 \mathrm{E}-03$ & 1.94 \\
& 64 & $2.56 \mathrm{E}-04$ & 2.04 & $7.85 \mathrm{E}-04$ & 1.99 & $2.56 \mathrm{E}-04$ & 2.04 & $7.85 \mathrm{E}-04$ & 1.99 \\
& 128 & $6.36 \mathrm{E}-05$ & 2.01 & $1.97 \mathrm{E}-04$ & 2.00 & $6.36 \mathrm{E}-05$ & 2.01 & $1.97 \mathrm{E}-04$ & 2.00 \\
& 256 & $1.59 \mathrm{E}-05$ & 2.00 & $4.92 \mathrm{E}-05$ & 2.00 & $1.59 \mathrm{E}-05$ & 2.00 & $4.92 \mathrm{E}-05$ & 2.00 \\
& 512 & $3.97 \mathrm{E}-06$ & 2.00 & $1.23 \mathrm{E}-05$ & 2.00 & $3.97 \mathrm{E}-06$ & 2.00 & $1.23 \mathrm{E}-05$ & 2.00 \\
\hline \multirow{6}{*}{$P^{2}$} & 16 & $1.95 \mathrm{E}-04$ & - & $3.11 \mathrm{E}-05$ & - & $1.95 \mathrm{E}-04$ & - & $3.11 \mathrm{E}-05$ & - \\
& 32 & $2.45 \mathrm{E}-05$ & 2.99 & $3.74 \mathrm{E}-06$ & 3.06 & $2.45 \mathrm{E}-05$ & 2.99 & $3.74 \mathrm{E}-06$ & 3.06 \\
& 64 & $3.07 \mathrm{E}-06$ & 3.00 & $4.63 \mathrm{E}-07$ & 3.01 & $3.07 \mathrm{E}-06$ & 3.00 & $4.63 \mathrm{E}-07$ & 3.01 \\
& 128 & $3.83 \mathrm{E}-07$ & 3.00 & $5.77 \mathrm{E}-08$ & 3.00 & $3.83 \mathrm{E}-07$ & 3.00 & $5.77 \mathrm{E}-08$ & 3.00 \\
& 256 & $4.79 \mathrm{E}-08$ & 3.00 & $7.21 \mathrm{E}-09$ & 3.00 & $4.79 \mathrm{E}-08$ & 3.00 & $7.21 \mathrm{E}-09$ & 3.00 \\
& 512 & $5.99 \mathrm{E}-09$ & 3.00 & $9.00 \mathrm{E}-10$ & 3.00 & $5.99 \mathrm{E}-09$ & 3.00 & $9.00 \mathrm{E}-10$ & 3.00 \\
\hline
\end{tabular}

TABLE 2. Errors and convergence orders of $p_{h}, q_{h}, r_{h}$ produced by the scheme (3.38) with $k=0,1,2,3$ in Example 4.2.

\begin{tabular}{clllllll}
\hline \hline \multirow{2}{*}{$N_{x} \times N_{y}$} & \multicolumn{2}{c}{$\left\|p-p_{h}\right\|$} & \multicolumn{2}{c}{$\left\|q-q_{h}\right\|$} & \multicolumn{2}{c}{$\left\|r-r_{h}\right\|$} \\
\cline { 3 - 8 } & & $L^{2}$ error & order & $L^{2}$ error & order & $L^{2}$ error & order \\
\hline \multirow{4}{*}{$P^{0}$} & $40 \times 16$ & $1.06 \mathrm{E}-01$ & - & $2.40 \mathrm{E}-02$ & - & $2.26 \mathrm{E}-02$ & - \\
& $80 \times 64$ & $5.36 \mathrm{E}-02$ & 0.98 & $1.04 \mathrm{E}-02$ & 1.21 & $1.01 \mathrm{E}-02$ & 1.16 \\
& $160 \times 128$ & $1.35 \mathrm{E}-02$ & 0.99 & $4.84 \mathrm{E}-03$ & 1.10 & $4.78 \mathrm{E}-03$ & 1.08 \\
& $20 \times 16$ & $8.85 \mathrm{E}-03$ & - & $6.92 \mathrm{E}-03$ & - & $8.64 \mathrm{E}-03$ & - \\
\multirow{3}{*}{$P^{1}$} & $40 \times 32$ & $2.22 \mathrm{E}-03$ & 1.99 & $1.95 \mathrm{E}-03$ & 1.83 & $2.42 \mathrm{E}-03$ & 1.84 \\
& $80 \times 64$ & $5.52 \mathrm{E}-04$ & 2.01 & $4.97 \mathrm{E}-04$ & 1.97 & $6.17 \mathrm{E}-04$ & 1.97 \\
& $160 \times 128$ & $1.37 \mathrm{E}-04$ & 2.01 & $1.25 \mathrm{E}-04$ & 2.00 & $1.55 \mathrm{E}-05$ & 1.99 \\
\hline \multirow{4}{*}{$P^{2}$} & $20 \times 16$ & $6.17 \mathrm{E}-04$ & - & $5.95 \mathrm{E}-04$ & - & $5.85 \mathrm{E}-04$ & - \\
& $40 \times 32$ & $7.22 \mathrm{E}-05$ & 3.10 & $9.68 \mathrm{E}-05$ & 2.62 & $9.64 \mathrm{E}-05$ & 2.60 \\
& $80 \times 64$ & $7.98 \mathrm{E}-06$ & 3.18 & $1.37 \mathrm{E}-05$ & 2.82 & $1.37 \mathrm{E}-05$ & 2.81 \\
& $160 \times 128$ & $1.01 \mathrm{E}-06$ & 2.98 & $1.77 \mathrm{E}-06$ & 2.96 & $1.77 \mathrm{E}-06$ & 2.96 \\
\hline \multirow{3}{*}{$P^{3}$} & $20 \times 16$ & $2.66 \mathrm{E}-05$ & - & $5.32 \mathrm{E}-05$ & - & $4.79 \mathrm{E}-05$ & - \\
& $40 \times 32$ & $1.80 \mathrm{E}-06$ & 3.89 & $5.42 \mathrm{E}-06$ & 3.30 & $4.39 \mathrm{E}-06$ & 3.45 \\
& $80 \times 64$ & $1.15 \mathrm{E}-07$ & 3.97 & $4.04 \mathrm{E}-07$ & 3.75 & $3.27 \mathrm{E}-07$ & 3.75 \\
& $160 \times 128$ & $7.18 \mathrm{E}-09$ & 4.00 & $2.61 \mathrm{E}-08$ & 3.95 & $2.11 \mathrm{E}-08$ & 3.95 \\
\hline
\end{tabular}


TABLE 3. Errors and convergence orders of $u_{h}, v_{h}, w_{h}$ produced by the scheme (3.38) with $k=0,1,2,3$ in Example 4.2.

\begin{tabular}{llllllll}
\hline \hline \multirow{2}{*}{$N_{x} \times N_{y}$} & \multicolumn{2}{c}{$\left\|p-u_{h}\right\|$} & \multicolumn{2}{c}{$\left\|q-v_{h}\right\|$} & \multicolumn{2}{c}{$\left\|r-w_{h}\right\|$} \\
\cline { 2 - 8 } & & $L^{2}$ error & order & $L^{2}$ error & order & $L^{2}$ error & order \\
\hline \multirow{4}{*}{$P^{0}$} & $20 \times 16$ & $1.06 \mathrm{E}-01$ & - & $2.40 \mathrm{E}-02$ & - & $2.26 \mathrm{E}-02$ & - \\
& $40 \times 32$ & $5.36 \mathrm{E}-02$ & 0.98 & $1.04 \mathrm{E}-02$ & 1.21 & $1.01 \mathrm{E}-02$ & 1.16 \\
& $80 \times 64$ & $2.69 \mathrm{E}-02$ & 0.99 & $4.84 \mathrm{E}-03$ & 1.10 & $4.78 \mathrm{E}-03$ & 1.08 \\
& $160 \times 128$ & $1.35 \mathrm{E}-02$ & 1.00 & $2.34 \mathrm{E}-03$ & 1.05 & $2.33 \mathrm{E}-03$ & 1.04 \\
\hline \multirow{4}{*}{$P^{1}$} & $20 \times 16$ & $8.85 \mathrm{E}-03$ & - & $6.92 \mathrm{E}-03$ & - & $8.64 \mathrm{E}-03$ & - \\
& $40 \times 32$ & $2.22 \mathrm{E}-03$ & 1.99 & $1.95 \mathrm{E}-03$ & 1.83 & $2.42 \mathrm{E}-03$ & 1.84 \\
& $80 \times 64$ & $5.52 \mathrm{E}-04$ & 2.01 & $4.97 \mathrm{E}-04$ & 1.97 & $6.17 \mathrm{E}-04$ & 1.97 \\
& $160 \times 128$ & $1.37 \mathrm{E}-04$ & 2.01 & $1.25 \mathrm{E}-04$ & 2.00 & $1.55 \mathrm{E}-05$ & 1.99 \\
\hline \multirow{4}{*}{$P^{2}$} & $20 \times 16$ & $6.17 \mathrm{E}-04$ & - & $5.95 \mathrm{E}-04$ & - & $5.85 \mathrm{E}-04$ & - \\
& $40 \times 32$ & $7.22 \mathrm{E}-05$ & 3.10 & $9.68 \mathrm{E}-05$ & 2.62 & $9.64 \mathrm{E}-05$ & 2.60 \\
& $80 \times 64$ & $7.98 \mathrm{E}-06$ & 3.18 & $1.37 \mathrm{E}-05$ & 2.82 & $1.37 \mathrm{E}-05$ & 2.81 \\
& $160 \times 128$ & $1.01 \mathrm{E}-06$ & 2.98 & $1.77 \mathrm{E}-06$ & 2.96 & $1.77 \mathrm{E}-06$ & 2.96 \\
\hline \multirow{3}{*}{$P^{3}$} & $20 \times 16$ & $2.66 \mathrm{E}-05$ & - & $5.32 \mathrm{E}-05$ & - & $4.79 \mathrm{E}-05$ & - \\
& $40 \times 32$ & $1.80 \mathrm{E}-06$ & 3.89 & $5.42 \mathrm{E}-06$ & 3.30 & $4.39 \mathrm{E}-06$ & 3.45 \\
& $80 \times 64$ & $1.15 \mathrm{E}-07$ & 3.97 & $4.04 \mathrm{E}-07$ & 3.75 & $3.27 \mathrm{E}-07$ & 3.75 \\
& $160 \times 128$ & $7.18 \mathrm{E}-09$ & 4.00 & $2.61 \mathrm{E}-08$ & 3.95 & $2.11 \mathrm{E}-08$ & 3.95 \\
\hline
\end{tabular}

solution decays very quickly with smaller $\alpha$, and the $L^{2}$ errors and energies of different $\alpha$ become flat as time evolves. For the $P^{1}$ element, the $L^{2}$ error increases slower and the energy decays slower when $\alpha=\sqrt{3} / 4$. This coincides with the theoretical result in (3.36) in the dispersion analysis, since the magnitude of the leading error term $i \frac{h^{3} k^{4}\left(3+16 \alpha^{2}\right)}{1152 \alpha}$ in (3.36) reaches its minimum at $\alpha=\sqrt{3} / 4$. For the $P^{2}$ element, the $L^{2}$ error and energy almost remain the same during the time evolution. This indicates our method is of low dissipation and very suitable for long time simulation, especially when the polynomial degree is high.

\section{Concluding Remarks}

In this paper, we propose the central DG method for solving second order wave equations. Instead of dealing with the scalar equation directly, we focus our attention on the first order system which is equivalent to the original equation. We construct the central DG scheme for the system, and derive the $L^{2}$ stability and the optimal error estimates. In the error estimates, we adopt the so-called shifting technique in [22] to construct a special local projection. The main difference between the projection here and the one in [22] is that the unknowns are highly coupled in the proposed scheme, making the analysis of the projection more difficult. Several lemmas and propositions are proposed to overcome this difficulty, and we have eventually obtained the the optimal error estimates for arbitrary $P^{k}$ polynomial space on uniform Cartesian meshes. We also perform a dispersion analysis for the proposed scheme in the one dimensional case, showing that the dissipation of the numerical solution with $P^{1}$ elements has a minimum with a suitable choice of the parameter $\alpha$. We present several numerical examples, and all of them coincide very well with our theoretical results. In the error estimates, there is an assumption that the mesh should be uniform so that the shifting technique could work. It is very challenging to remove this restriction and this will be one of our future works. Besides, the extension to nonlinear equations and the dispersion analysis for general $P^{k}$ elements are also very intriguing and challenging that constitute our future work. 


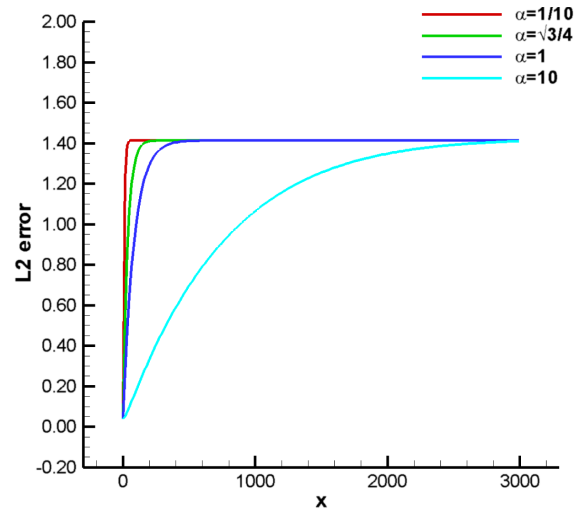

(a)

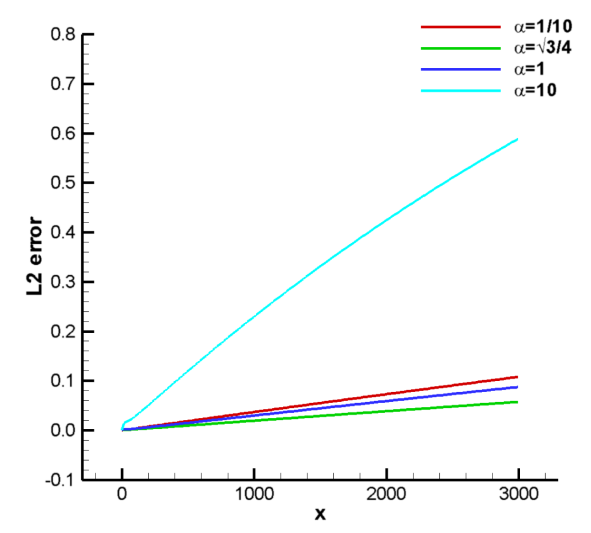

(c)

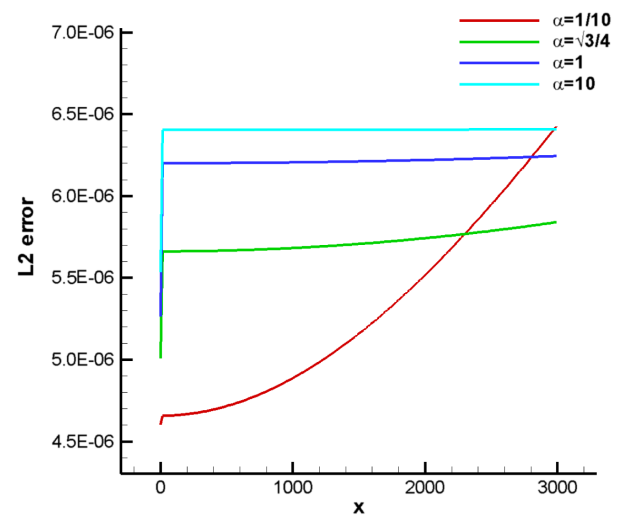

(e)

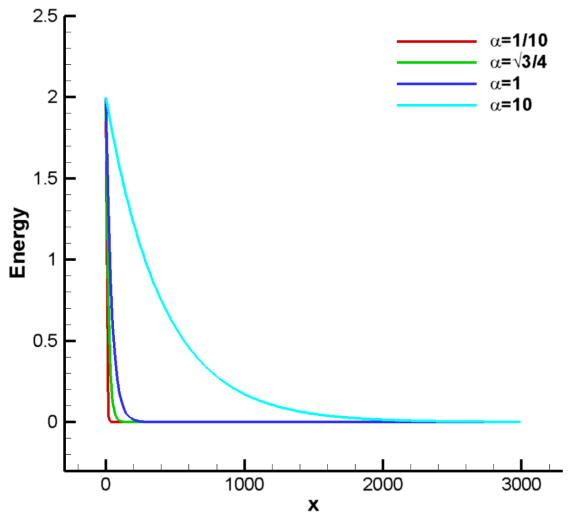

(b)

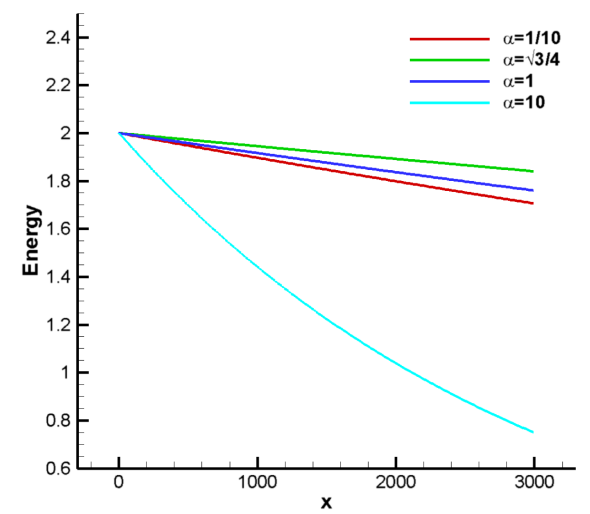

(d)

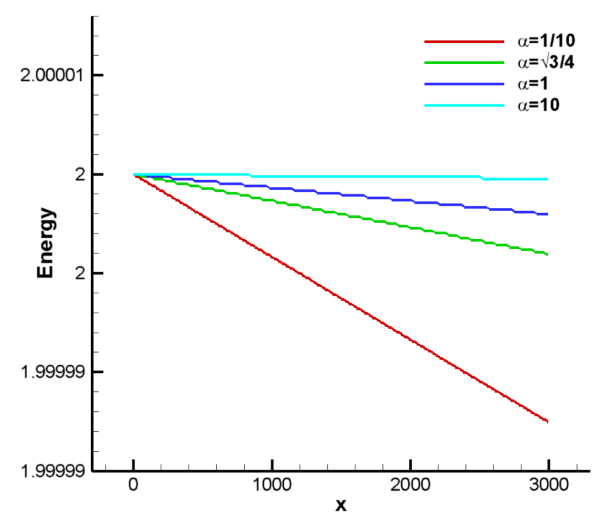

(f)

Figure 2. $L^{2}$ error and energy evolve against time by the DG scheme (3.2) with $P^{0}, P^{1}$ and $P^{2}$ element. The spatial mesh size $h=\pi / 32 . \tau_{\max }=\alpha h, \alpha=0.1, \sqrt{3} / 4,1,10$. The final time is $T=3000$. (a) $L^{2}$ error, $P^{0}$ element. (b) Energy, $P^{0}$ element. (c) $L^{2}$ error, $P^{1}$ element. (d) Energy, $P^{1}$ element. (e) $L^{2}$ error, $P^{2}$ element. (f) Energy, $P^{2}$ element. 


\section{Appendix A. Proof of a feW technical lemmas and propositions}

In this appendix, we collect the proof of some of the technical lemmas and propositions in the error estimates.

\section{A.1. Proof of Lemma 3.2}

Proof. Note that $\left(\mathbb{P}_{h}^{1, \star} p, \mathbb{P}_{h}^{2, \star} q\right)$ belongs to the finite dimensional space $\left[P^{k}([-1,1])\right]^{2}$, thus we only need to solve a linear system to obtain $\left(\mathbb{P}_{h}^{1, \star} p, \mathbb{P}_{h}^{2, \star} q\right)$ and the existence and uniqueness are equivalent. Thus, we only prove the uniqueness of the projection $\mathbb{P}_{h}^{\star}$. We set $\left(p_{I}, q_{I}\right)=\left(\mathbb{P}_{h}^{1, \star} p, \mathbb{P}_{h}^{2, \star} q\right)$ with $p=q=0$, and would like to prove $\left(p_{I}, q_{I}\right)=(0,0)$. By the definition of the projection $\mathbb{P}_{h}^{\star}$, we take $\varphi_{h}=p_{I}$ and $\bar{\varphi}_{h}=q_{I}$ in $\widetilde{P_{h}}\left(p_{I}, q_{I} ; \varphi_{h}\right)$ and $\widetilde{P_{h}}\left(q_{I}, p_{I} ; \bar{\varphi}_{h}\right)$, and do a change of variables $x \rightarrow x+1$ to obtain

$$
\begin{aligned}
0= & \widetilde{P_{h}}\left(p_{I}, q_{I} ; p_{I}\right)+\widetilde{P_{h}}\left(q_{I}, p_{I} ; q_{I}\right) \\
= & \frac{1}{2 \alpha}\left(\int_{-1}^{0} p_{I}(x+1) p_{I}(x) \mathrm{d} x+\int_{0}^{1} p_{I}(x-1) p_{I}(x) \mathrm{d} x-\int_{-1}^{1} p_{I}(x) p_{I}(x) \mathrm{d} x\right) \\
& +\frac{1}{2 \alpha}\left(\int_{-1}^{0} q_{I}(x+1) q_{I}(x) \mathrm{d} x+\int_{0}^{1} q_{I}(x-1) q_{I}(x) \mathrm{d} x-\int_{-1}^{1} q_{I}(x) q_{I}(x) \mathrm{d} x\right) \\
& +\int_{-1}^{0} q_{I}(x+1)\left(p_{I}(x)\right)_{x} \mathrm{~d} x+\int_{0}^{1} q_{I}(x-1)\left(p_{I}(x)\right)_{x} \mathrm{~d} x \\
& +\int_{-1}^{0} p_{I}(x+1)\left(q_{I}(x)\right)_{x} \mathrm{~d} x+\int_{0}^{1} p_{I}(x-1)\left(q_{I}(x)\right)_{x} \mathrm{~d} x \\
& -q_{I}(0)\left(p_{I}(1)-q_{I}(-1)\right)-p_{I}(0)\left(q_{I}(1)-q_{I}(-1)\right) \\
= & \frac{1}{2 \alpha}\left(\int_{0}^{1} 2 p_{I}(x) p_{I}(x-1) \mathrm{d} x-\int_{0}^{1} p_{I}(x) p_{I}(x) \mathrm{d} x-\int_{0}^{1} p_{I}(x-1) p_{I}(x-1) \mathrm{d} x\right) \\
& +\frac{1}{2 \alpha}\left(\int_{0}^{1} 2 q_{I}(x) q_{I}(x-1) \mathrm{d} x-\int_{0}^{1} q_{I}(x) q_{I}(x) \mathrm{d} x-\int_{0}^{1} q_{I}(x-1) q_{I}(x-1) \mathrm{d} x\right) \\
& +\int_{0}^{1}\left(q_{I}(x) p_{I}(x-1)\right)_{x} \mathrm{~d} x+\int_{0}^{1}\left(q_{I}(x-1) p_{I}(x)\right)_{x} \mathrm{~d} x \\
& -q_{I}(0)\left(p_{I}(1)-q_{I}(-1)\right)-p_{I}(0)\left(q_{I}(1)-q_{I}(-1)\right) \\
= & -\frac{1}{2 \alpha} \int_{0}^{1}\left(p_{I}(x-1)-p_{I}(x)\right)^{2} \mathrm{~d} x-\frac{1}{2 \alpha} \int_{0}^{1}\left(q_{I}(x-1)-q_{I}(x)\right)^{2} \mathrm{~d} x .
\end{aligned}
$$

Then it indicates that

$$
p_{I}(x)=p_{I}(x-1), \quad q_{I}(x)=q_{I}(x-1), \quad \forall x \in(0,1),
$$

which implies $p_{I}(x) \equiv$ constant and $q_{I}(x) \equiv$ constant. These together with (3.10a) and (3.10b), we have

$$
\left(p_{I}(x), q_{I}(x)\right) \equiv(0,0) .
$$

Thus we finish the proof of uniqueness. We now proceed to the proof of (3.14). We denote

$$
\left(p_{I}, q_{I}\right)=\left(\sum_{i=1}^{k+1} a_{i} x^{i-1}, \sum_{i=1}^{k+1} b_{i} x^{i-1}\right) .
$$


Take the test functions $\varphi_{h}=\bar{\varphi}_{h}=x^{i-1}, i=2, \ldots, k+1$, then we have

$$
\begin{aligned}
\widetilde{P_{h}}\left(p_{I}, q_{I} ; x^{i-1}\right) & =\sum_{\ell=1}^{k+1} D_{i, \ell} a_{\ell}+\sum_{\ell=1}^{k+1} D_{i, \ell+k+1} b_{\ell} \\
\widetilde{P_{h}}\left(q_{I}, p_{I} ; x^{i-1}\right) & =\sum_{\ell=1}^{k+1} D_{i+k+1, \ell} a_{\ell}+\sum_{\ell=1}^{k+1} D_{i+k+1, \ell+k+1} b_{\ell} \\
\int_{-1}^{1} p_{I} \mathrm{~d} x & =\sum_{\ell=1}^{k+1} D_{1, \ell} a_{\ell}, \quad \int_{-1}^{1} q_{I} \mathrm{~d} x=\sum_{\ell=1}^{k+1} D_{k+2, \ell+k+1} b_{\ell} .
\end{aligned}
$$

It is easy to prove

$$
\left|\widetilde{P_{h}}\left(p_{I}, q_{I} ; x^{i-1}\right)\right| \lesssim\|p\|_{\infty}+\|q\|_{\infty}, \quad\left|\widetilde{P_{h}}\left(q_{I}, p_{I} ; x^{i-1}\right)\right| \lesssim\|p\|_{\infty}+\|q\|_{\infty} .
$$

We denote

$$
\begin{aligned}
\sigma & =\left(a_{1}, \ldots, a_{k+1}, b_{1}, \ldots, b_{k+1}\right)^{T}, \\
\gamma_{1} & =\int_{-1}^{1} p \mathrm{~d} x, \quad \gamma_{i}=\widetilde{P_{h}}\left(p_{I}, q_{I} ; x^{i-1}\right), i=2, \ldots, k+1, \\
\gamma_{k+2} & =\int_{-1}^{1} q \mathrm{~d} x, \quad \gamma_{i+k+1}=\widetilde{P_{h}}\left(q_{I}, p_{I} ; x^{i-1}\right), i=2, \ldots, k+1 .
\end{aligned}
$$

We now obtain the following linear system:

$$
D \sigma=\gamma,
$$

and we can solve this linear system to get $\sigma=D^{-1} \gamma$. Each component of $\sigma$ is bounded by $\|p\|_{\infty}+\|q\|_{\infty}$, i.e. $\left|a_{i}\right| \lesssim\|p\|_{\infty}+\|q\|_{\infty}$ and $\left|b_{i}\right| \lesssim\|p\|_{\infty}+\|q\|_{\infty}, i=1, \ldots, k+1$. Therefore (3.14) holds true.

\section{A.2. Proof of Lemma 3.3}

Proof. We only prove one case that $\forall x \in\left[x_{j-\frac{1}{2}}, x_{j+\frac{1}{2}}\right]$,

$$
\begin{aligned}
a x^{k+1}-\mathbb{P}_{h}^{1, \star} p(x) & =a(x-h / 2)^{k+1}-\mathbb{Q}_{h}^{1, \star \star} p(x-h / 2), \\
b x^{k+1}-\mathbb{P}_{h}^{2, \star} q(x) & =b(x-h / 2)^{k+1}-\mathbb{Q}_{h}^{2, \star} q(x-h / 2) .
\end{aligned}
$$

We denote $\left(p_{I}, q_{I}\right) \in\left[P^{k}\left(I_{j}\right)\right]^{2}$ that

$$
\begin{aligned}
& p_{I}(x)=a x^{k+1}-a(x-h / 2)^{k+1}+\mathbb{Q}_{h}^{1, \star} p(x-h / 2), \\
& q_{I}(x)=b x^{k+1}-b(x-h / 2)^{k+1}+\mathbb{Q}_{h}^{2, \star} q(x-h / 2) .
\end{aligned}
$$

By the uniqueness of the projections, we only need to check $\left(p_{I}, q_{I}\right)$ satisfying (3.10a)-(3.10d). It is easy to verify that

$$
\begin{aligned}
\int_{I_{j}} p_{I} \mathrm{~d} x & =\int_{I_{j}} a x^{k+1} \mathrm{~d} x-\int_{I_{j}} a(x-h / 2)^{k+1}-\mathbb{Q}_{h}^{1, \star} p(x-h / 2) \mathrm{d} x \\
& =\int_{I_{j}} a x^{k+1} \mathrm{~d} x-\int_{I_{j-\frac{1}{2}}} a x^{k+1}-\mathbb{Q}_{h}^{1, \star} p(x) \mathrm{d} x \\
& =\int_{I_{j}} a x^{k+1} \mathrm{~d} x=\int_{I_{j}} p \mathrm{~d} x,
\end{aligned}
$$


where we use the definition of projection $\mathbb{Q}_{h}^{1, \star}$ in $(3.12 \mathrm{a})$ on $I_{j-\frac{1}{2}}$. By the same argument above, we have

$$
\int_{I_{j}} q_{I} d=\int_{I_{j}} b x^{k+1} \mathrm{~d} x=\int_{I_{j}} q \mathrm{~d} x .
$$

And, $\forall \varphi_{h}(x) \in P^{k}\left(I_{j}\right)$ we have

$$
\begin{aligned}
\widetilde{P_{h}}\left(p_{I}, q_{I} ; \varphi_{h}\right)_{j} & =\widetilde{P_{h}}\left(p, q ; \varphi_{h}\right)_{j}-\widetilde{P_{h}}\left(p(x-h / 2)-\mathbb{Q}_{h}^{1, \star} p(x-h / 2), q(x-h / 2)-\mathbb{Q}_{h}^{2, \star} q(x-h / 2) ; \varphi_{h}\right)_{j} \\
& =\widetilde{P_{h}}\left(p, q ; \varphi_{h}\right)_{j}-\widetilde{Q_{h}}\left(p(x)-\mathbb{Q}_{h}^{1, \star} p(x), q(x)-\mathbb{Q}_{h}^{2, \star} q(x) ; \varphi_{h}(x+h / 2)\right)_{j-\frac{1}{2}} \\
& =\widetilde{P_{h}}\left(p, q ; \varphi_{h}\right)_{j},
\end{aligned}
$$

where we have used the fact that $\varphi_{h}(x+h / 2) \in P^{k}\left(I_{j-\frac{1}{2}}\right)$. By similar argument, we have

$$
\widetilde{P_{h}}\left(q_{I}, p_{I} ; \bar{\varphi}_{h}\right)_{j}=\widetilde{P_{h}}\left(q, p ; \bar{\varphi}_{h}\right)_{j}, \quad \forall \bar{\varphi}_{h}(x) \in P^{k}\left(I_{j}\right) .
$$

Therefore, the uniqueness of the projection $\mathbb{Q}_{h}^{\star}$ implies that $\left(p_{I}, q_{I}\right)=\left(\mathbb{P}_{h}^{1, \star} p, \mathbb{P}_{h}^{2, \star} q\right)$.

\section{A.3. Proof of Proposition 3.4}

Proof. Note that the projection preserves the $k$-th degree polynomials, which means when $(p, q) \in\left[P^{k}\left(K_{j}\right)\right]^{2}$, then $\left(p_{I}, q_{I}\right)=(p, q) \in\left[V_{h}\right]^{2}$ and $\left(r_{I}, s_{I}\right)=(p, q) \in\left[W_{h}\right]^{2}$. Thus we only need to consider the case $(p, q)=$ $\left(a x^{k+1}, b x^{k+1}\right)$, where $a, b$ are constants. For simplicity, we only prove (3.18), and other cases can be obtained by similar argument.

By Lemma 3.3, we have

$$
\begin{aligned}
B_{1}\left(r_{I}, p_{I}, s_{I} ; \varphi_{h}\right)_{j}= & \frac{1}{\tau_{\max }}\left(\int_{x_{j-\frac{1}{2}}}^{x_{j}} r_{I} \varphi_{h} \mathrm{~d} x+\int_{x_{j}}^{x_{j+\frac{1}{2}}} r_{I} \varphi_{h} \mathrm{~d} x-\int_{I_{j}} p_{I} \varphi_{h} \mathrm{~d} x\right) \\
& +\int_{x_{j-\frac{1}{2}}}^{x_{j}} s_{I} \partial_{x} \varphi_{h} \mathrm{~d} x+\int_{x_{j}}^{x_{j+\frac{1}{2}}} s_{I} \partial_{x} \varphi_{h} \mathrm{~d} x \\
& -s_{I}\left(x_{j+\frac{1}{2}}\right) \varphi_{h}\left(x_{j+\frac{1}{2}}^{-}\right)+s_{I}\left(x_{j-\frac{1}{2}}\right) \varphi_{h}\left(x_{j-\frac{1}{2}}^{+}\right) \\
= & \frac{1}{\tau_{\max }}\left(\int_{x_{j-\frac{1}{2}}}^{x_{j}}\left(p_{I}(x+h / 2)-p(x+h / 2)+p(x)\right) \varphi_{h} \mathrm{~d} x\right. \\
& \left.+\int_{x_{j}}^{x_{j+\frac{1}{2}}}\left(p_{I}(x-h / 2)-p(x-h / 2)+p(x)\right) \varphi_{h} \mathrm{~d} x-\int_{I_{j}} p_{I} \varphi_{h} \mathrm{~d} x\right) \\
& +\int_{x_{j-\frac{1}{2}}}^{x_{j}}\left(q_{I}(x+h / 2)-q(x+h / 2)+q(x)\right) \partial_{x} \varphi_{h} \mathrm{~d} x \\
& +\int_{x_{j}}^{x_{j+\frac{1}{2}}}\left(q_{I}(x-h / 2)-q(x-h / 2)+q(x)\right) \partial_{x} \varphi_{h} \mathrm{~d} x \\
& -\left(q_{I}\left(x_{j}\right)-q\left(x_{j}\right)+q\left(x_{j+\frac{1}{2}}\right)\right) \varphi_{h}\left(x_{j+\frac{1}{2}}^{-}\right) \\
& +\left(q_{I}\left(x_{j}\right)-q\left(x_{j}\right)+q\left(x_{j-\frac{1}{2}}\right)\right) \varphi_{h}\left(x_{j-\frac{1}{2}}^{+}\right) \\
= & \widetilde{P}_{h}\left(p_{I}-p, q_{I}-q ; \varphi_{h}\right)_{j}+B_{1}\left(p, p, q ; \varphi_{h}\right)_{j} \\
= & B_{1}\left(p, p, q ; \varphi_{h}\right)_{j} . \\
&
\end{aligned}
$$


Then we obtain the desired result.

\section{A.4. Proof of Lemma 3.8}

Proof. Similar to the proof of Lemma 3.2, we assume that $(p, q, r)=(0,0,0)$. For simplicity, we take $\left(p_{I}, q_{I}, r_{I}\right)=$ $\left(\mathbb{P}_{h}^{1, \star} p, \mathbb{P}_{h}^{2, \star} q, \mathbb{P}_{h}^{3, \star} r\right)$, and $\left(\varphi_{h}, \bar{\varphi}_{h}, \tilde{\varphi}_{h}\right)=\left(p_{I}, q_{I}, r_{I}\right)$ in $(3.45 \mathrm{~d})-(3.45 \mathrm{f})$. To simplify the formulation, we transform all the integration regions into the same subcell $K_{i, j}^{1}=\left[x_{i-\frac{1}{2}}, x_{i}\right] \times\left[y_{j-\frac{1}{2}}, y_{j}\right]$ with a change of variables. Then we have

$$
\begin{aligned}
0= & \widetilde{P}_{h}^{1}\left(p_{I}, q_{I}, r_{I} ; p_{I}\right)_{i, j}+{\widetilde{P_{h}}}^{2}\left(q_{I}, p_{I} ; q_{I}\right)_{i, j}+{\widetilde{P_{h}}}^{2}\left(r_{I}, p_{I} ; r_{I}\right)_{i, j} \\
= & \frac{-1}{\tau_{\max }}\left(\int_{K_{i, j}^{1}}\left(p_{I}\left(x+h_{x} / 2, y+h_{y} / 2\right)-p_{I}(x, y)\right)^{2} \mathrm{~d} x \mathrm{~d} y\right. \\
& \left.+\int_{K_{i, j}^{1}}\left(p_{I}\left(x+h_{x} / 2, y\right)-p_{I}\left(x, y+h_{y} / 2\right)\right)^{2} \mathrm{~d} x \mathrm{~d} y\right) \\
& +\frac{-1}{\tau_{\max }}\left(\int_{K_{i, j}^{1}}\left(q_{I}\left(x+h_{x} / 2, y+h_{y} / 2\right)-q_{I}(x, y)\right)^{2} \mathrm{~d} x \mathrm{~d} y\right. \\
& \left.+\int_{K_{i, j}^{1}}\left(q_{I}\left(x+h_{x} / 2, y\right)-q_{I}\left(x, y+h_{y} / 2\right)\right)^{2} \mathrm{~d} x \mathrm{~d} y\right) \\
+ & \frac{-1}{\tau_{\max }}\left(\int_{K_{i, j}^{1}}\left(r_{I}\left(x+h_{x} / 2, y+h_{y} / 2\right)-r_{I}(x, y)\right)^{2} \mathrm{~d} x \mathrm{~d} y\right. \\
& \left.+\int_{K_{i, j}^{1}}\left(r_{I}\left(x+h_{x} / 2, y\right)-r_{I}\left(x, y+h_{y} / 2\right)\right)^{2} \mathrm{~d} x \mathrm{~d} y\right),
\end{aligned}
$$

which implies that $\forall(x, y) \in K_{i, j}^{1}$, we have

$$
\begin{array}{ll}
p_{I}(x, y)=p_{I}\left(x+h_{x} / 2, y+h_{y} / 2\right), & p_{I}\left(x+h_{x} / 2, y\right)=p_{I}\left(x, y+h_{y} / 2\right) \\
q_{I}(x, y)=q_{I}\left(x+h_{x} / 2, y+h_{y} / 2\right), & q_{I}\left(x+h_{x} / 2, y\right)=q_{I}\left(x, y+h_{y} / 2\right) \\
r_{I}(x, y)=r_{I}\left(x+h_{x} / 2, y+h_{y} / 2\right), & r_{I}\left(x+h_{x} / 2, y\right)=r_{I}\left(x, y+h_{y} / 2\right)
\end{array}
$$

Thus $p_{I}(x, y), q_{I}(x, y), r_{I}(x, y)$ are constants on $K_{i, j}$. By $(3.45 \mathrm{a})-(3.45 \mathrm{c})$, we immediately get $p_{I}=q_{I}=r_{I}=0$. We have finished the proof of uniqueness, which is equivalent to the existence. Notice that the projection is a local projection, hence we can do a change of variables to transform the integration regions into the reference cell $[-1,1]^{2}$ by taking $\xi=2\left(x-x_{i}\right) / h$ and $\eta=2\left(y-y_{j}\right) / h$. Taking a similar derivation as in A.1, we have

$$
\left\|p_{I}\right\|_{\infty}+\left\|q_{I}\right\|_{\infty}+\left\|r_{I}\right\|_{\infty} \leq C(k)\left(\|p\|_{\infty}+\|q\|_{\infty}+\|r\|_{\infty}\right) .
$$

\section{A.5. Proof of Lemma 3.9}

Proof. We only give the proof of one of the cases, as the others can be verified by similar arguments. We will prove the first equality of (3.48). We denote

$$
\begin{aligned}
& p_{I}(x, y)=p(x, y)-p\left(x-h_{x} / 2, y-h_{y} / 2\right)+\mathbb{Q}_{h}^{1, \star} p\left(x-h_{x} / 2, y-h_{y} / 2\right), \\
& q_{I}(x, y)=q(x, y)-q\left(x-h_{x} / 2, y-h_{y} / 2\right)+\mathbb{Q}_{h}^{2, \star} q\left(x-h_{x} / 2, y-h_{y} / 2\right), \\
& r_{I}(x, y)=r(x, y)-r\left(x-h_{x} / 2, y-h_{y} / 2\right)+\mathbb{Q}_{h}^{3, \star} r\left(x-h_{x} / 2, y-h_{y} / 2\right) .
\end{aligned}
$$


Note that $\left(p_{I}, q_{I}, r_{I}\right) \in\left[P^{k}\left(K_{i, j}\right)\right]^{3}$, by the uniqueness of the projection $\mathbb{P}_{h}^{\star}$, we only need to verify that $\left(p_{I}, q_{I}, r_{I}\right)$ satisfies the definition of the projection $\mathbb{P}_{h}^{\star}$. It is easy to check

$$
\begin{aligned}
\int_{K_{i, j}} p_{I}(x, y) \mathrm{d} x \mathrm{~d} y & =\int_{K_{i, j}} p(x, y) \mathrm{d} x \mathrm{~d} y-\int_{K_{i-\frac{1}{2}, j-\frac{1}{2}}}\left(p(x, y)-\mathbb{Q}_{h}^{\star} p(x, y)\right) \mathrm{d} x \mathrm{~d} y \\
& =\int_{K_{i, j}} p(x, y) \mathrm{d} x \mathrm{~d} y .
\end{aligned}
$$

Thus (3.45a) is verified. Similarly, (3.45b)-(3.45c) can also be verified. For (3.45d), we have

$$
\begin{aligned}
\widetilde{P}_{h}^{1}\left(p_{I}, q_{I}, r_{I} ; \varphi_{h}\right)_{i, j}= & {\widetilde{P_{h}}}^{1}\left(p, q, r ; \varphi_{h}\right)_{i, j} \\
& -{\widetilde{P_{h}}}^{1}\left(p\left(x-h_{x} / 2, y-h_{y} / 2\right)-\mathbb{Q}_{h}^{1, \star} p\left(x-h_{x} / 2, y-h_{y} / 2\right),\right. \\
& q\left(x-h_{x} / 2, y-h_{y} / 2\right)-\mathbb{Q}_{h}^{2, \star} q\left(x-h_{x} / 2, y-h_{y} / 2\right), \\
\quad & \left.\quad\left(x-h_{x} / 2, y-h_{y} / 2\right)-\mathbb{Q}_{h}^{3, \star} r\left(x-h_{x} / 2, y-h_{y} / 2\right) ; \varphi_{h}\right)_{i, j} \\
= & \widetilde{P}_{h}^{1}\left(p, q, r ; \varphi_{h}\right)_{i, j} \\
& -{\widetilde{Q_{h}}}^{1}\left(p(x, y)-\mathbb{Q}_{h}^{1, \star} p(x, y), q(x, y)-\mathbb{Q}_{h}^{2, \star \star} q(x, y),\right. \\
\quad & \left.\quad r(x, y)-\mathbb{Q}_{h}^{3, \star} r(x, y) ; \varphi_{h}\left(x+h_{x} / 2, y+h_{y} / 2\right)\right)_{i-\frac{1}{2}, j-\frac{1}{2}} \\
= & \widetilde{P}_{h}^{1}\left(p, q, r ; \varphi_{h}\right)_{i, j} .
\end{aligned}
$$

Thus (3.45d) is verified. Also, (3.45e)-(3.45f) can be verified in the similar way.

\section{A.6. Proof of Lemma 3.10}

Proof. We only prove (3.49) in the following. Using Lemma 3.9, we have

$$
\begin{aligned}
\mathcal{B}_{1}\left(u_{I}, p_{I}, v_{I}, w_{I} ; \varphi_{h}\right)_{i, j} & =\mathcal{B}_{1}\left(p, p, q, r ; \varphi_{h}\right)_{i, j}+\widetilde{P}_{h}^{1}\left(p_{I}-p, q_{I}-q, r_{I}-r ; \varphi_{h}\right)_{i, j} \\
& =\mathcal{B}_{1}\left(p, p, q, r ; \varphi_{h}\right)_{i, j} .
\end{aligned}
$$

(3.50)-(3.54) can be obtained by similar argument.

Acknowledgements. Research of Yong Liu was supported by the China Scholarship Council and the fellowship of China Postdoctoral Science Foundation No. 2020TQ0343. Jianfang Lu was supported by NSFC grant 11901213. Chi-Wang Shu was supported by NSF grants DMS-1719410 and DMS-2010107, and AFOSR grant FA9550-20-1-0055. Mengping Zhang was supported by NSFC grant 11871448.

\section{REFERENCES}

[1] M. Baccouch, A local discontinuous Galerkin method for the second-order wave equation. Comput. Methods Appl. Mech. Eng. 209 (2012) 129-143.

[2] J.C. Butcher, Numerical Methods for Ordinary Differential Equations, 2nd edition. John Wiley \& Sons (2008).

[3] Y. Cheng, C.S. Chou, F. Li and Y. Xing, $L^{2}$ stable discontinuous Galerkin methods for one dimensional two-way wave equations. Math. Comput. 86 (2017) 121-155.

[4] E.T. Chung and B. Engquist, Optimal discontinuous Galerkin methods for the acoustic wave equation in higher dimensions. SIAM J. Numer. Anal. 47 (2009) 3820-3848.

[5] P.G. Ciarlet, The Finite Element Method for Elliptic Problems. North Holland (1978).

[6] B. Cockburn and C.-W. Shu, TVB Runge-Kutta local projection discontinuous Galerkin finite element method for conservation laws. II. general framework. Math. Comput. 52 (1989) 411-435.

[7] B. Cockburn and C.-W. Shu, The Runge-Kutta local projection P1-discontinuous Galerkin finite element method for scalar conservation laws. Math. Model. Numer. Anal. 25 (1991) 337-361. 
[8] B. Cockburn and C.-W. Shu, The Runge-Kutta discontinuous Galerkin method for conservation laws V: multidimensional systems. J. Comput. Phys. 141 (1998) 199-224.

[9] B. Cockburn, S.-Y. Lin and C.-W. Shu, TVB Runge-Kutta local projection discontinuous Galerkin finite element method for conservation laws III: one dimensional systems. J. Comput. Phys. 84 (1989) 90-113.

[10] B. Cockburn, S. Hou and C.-W. Shu, The Runge-Kutta local projection discontinuous Galerkin finite element method for conservation laws IV: the multidimensional case. Math. Comput. 54 (1990) 545-581.

[11] B. Cockburn, G. Kanschat, I. Perugia and D. Schötzau, Superconvergence of the local discontinuous Galerkin method for elliptic problems on Cartesian grids. SIAM J. Numer. Anal. 39 (2001) 264-285.

[12] G.C. Cohen, Higher-order numerical methods for transient wave equations. In: Scientific Computation. Springer-Verlag, Berlin (2002). With a foreword by R. Glowinski.

[13] D.R. Durran, Numerical methods for wave equations in geophysical fluid dynamics. In: Vol. 32 of Texts in Applied Mathematics. Springer, New York (1999).

[14] D. Gottlieb and J.S. Hesthaven, Spectral methods for hyperbolic problems. J. Comput. Appl. Math. 128 (2001) 83-131.

[15] D. Gottlieb and S.A. Orszag, Numerical analysis of spectral methods: theory and applications. In: CBMS-NSF Regional Conference Series in Applied Mathematics, No. 26. Society for Industrial and Applied Mathematics, Philadelphia, PA (1977).

[16] S. Gottlieb, C.-W. Shu and E. Tadmor, Strong stability-preserving high-order time discretization methods. SIAM Rev. 43 (2001) 89-112.

[17] M.J. Grote, A. Schneebeli and D. Schötzau, Discontinuous Galerkin finite element method for the wave equation. SIAM J. Numer. Anal. 44 (2006) 2408-2431.

[18] N.A. Kampanis, J. Ekaterinaris and V. Dougalis, Effective Computational Methods for Wave Propagation. Chapman \& Hall/CRC (2008).

[19] R.J. LeVeque, Finite Volume Methods for Hyperbolic Problems. Cambridge Texts in Applied Mathematics. Cambridge University Press, Cambridge (2002).

[20] Y. Liu, C.-W. Shu, E. Tadmor and M. Zhang, Central discontinuous Galerkin methods on overlapping cells with a nonoscillatory hierarchical reconstruction. SIAM J. Numer. Anal. 45 (2007) 2442-2467.

[21] Y. Liu, C.-W. Shu, E. Tadmor and M. Zhang, $L^{2}$ stability analysis of the central discontinuous Galerkin method and a comparison between the central and regular discontinuous Galerkin methods. ESAIM: M2AN 42 (2008) 593-607.

[22] Y. Liu, C.-W. Shu and M. Zhang, Optimal error estimates of the semidiscrete central discontinuous Galerkin methods for linear hyperbolic equations. SIAM J. Numer. Anal. 56 (2018) 520-541.

[23] Y. Liu, C.-W. Shu and M. Zhang, Optimal error estimates of the semidiscrete discontinuous Galerkin methods for two dimensional hyperbolic equations on Cartesian meshes using $P^{k}$ elements. ESAIM: M2AN 54 (2020) 705-726.

[24] X. Meng, C.-W. Shu and B. Wu, Optimal error estimates for discontinuous Galerkin methods based on upwind-biased fluxes for linear hyperbolic equations. Math. Comput. 85 (2016) 1225-1261.

[25] W.H. Reed and T.R. Hill, Triangular mesh methods for the neutron transport equation. Los Alamos Scientific Laboratory report LA-UR-73-479 (1973).

[26] M.A. Reyna and F. Li, Operator bounds and time step conditions for DG and central DG methods. J. Sci. Comput. 62 (2015) $532-554$.

[27] Y. Xing, C.-S. Chou and C.-W. Shu, Energy conserving local discontinuous Galerkin methods for wave propagation problems. Inverse Prob. Imaging 7 (2013) 967-986.

[28] Y. Xu and C.-W. Shu, Optimal error estimates of the semidiscrete local discontinuous galerkin methods for high order wave equations. SIAM J. Numer. Anal. 50 (2012) 79-104.

[29] Z. Xu and Y. Liu, New central and central discontinuous Galerkin schemes on overlapping cells of unstructured grids for solving ideal magnetohydrodynamic equations with globally divergence-free magnetic field. J. Comput. Phys. 327 (2016) $203-224$.

[30] Y. Yang, X. Cai and J.-M. Qiu, Optimal convergence and superconvergence of semi-Lagrangian discontinuous Galerkin methods for linear convection equations in one space dimension. Math. Comput. 89 (2020) 2113-2139. 